\title{
Housing expenditure
}




\section{Background}

This chapter presents housing-related costs such as rent, mortgage payments, repair and maintenance, and home improvements. The first section outlines the definitions of housing expenditure: the Classification Of Individual COnsumption by Purpose (COICOP) definition, followed by the definition used in the analysis of this chapter, which includes expenditure not present in COICOP. This chapter also examines housing expenditure over time and by income, region, and household characteristics. The final section explores housing costs for renters, and for mortgage holders in more depth.

\section{Definitions of housing expenditure}

The COICOP system has been used to classify expenditure on the Living Costs and Food Survey (LCF) and previously the Expenditure and Food Survey (EFS) since 2001/02. COICOP is an internationally agreed system of classification for reporting consumption expenditure within National Accounts and is used by other household budget surveys across the European Union. Further information on COICOP can be found on the United Nations Statistics Division website: http://unstats.un.org/unsd/cr/registry/regct.asp? Lg=1.

Under COICOP, household consumption expenditure is categorised into the following 12 headings:

1. Food \& non-alcoholic drinks

2. Alcoholic drinks, tobacco \& narcotics

3. Clothing \& footwear

4. Housing (net), fuel \& power

5. Household goods \& services

6. Health

7. Transport

8. Communication

9. Recreation \& culture

10. Education

11. Restaurants \& hotels

12. Miscellaneous goods \& services

It is important to note that COICOP classified housing costs do not include what is considered to be non-consumption expenditure, for example, mortgage interest, mortgage capital repayments, mortgage protection premiums, council tax and domestic rates.

In addition to the 12 COICOP expenditure categories, the tables contained in Appendix A include a category called 'other expenditure items' under which certain non-consumption expenditures can be found. This category includes the following housing-related costs: mortgage interest payments; mortgage protection premiums; council tax; and domestic rates. Housing costs that are not included in either the COICOP definition of housing or the 'other expenditure item' category are captured within the 'other items recorded' category that can be viewed in Table A1 in Appendix A.

For the purpose of this chapter all data relating to housing expenditure have been combined to facilitate an understanding of total housing costs. This comprehensive definition of housing expenditure is made up from three types of expenditure detailed in Table 2.1: expenditure included in COICOP, housing costs included in the 'other expenditure items' and 'other items recorded' categories of Family Spending. 
It should also be noted that throughout Family Spending, including this chapter, rent excluding service charges and benefit receipts associated with housing (net rent) has been used when calculating total expenditure. This convention ensures that rebates, benefits and allowances are excluded from the calculation of total household expenditure on rent.

\section{Table 2.1 Definition of total housing expenditure}

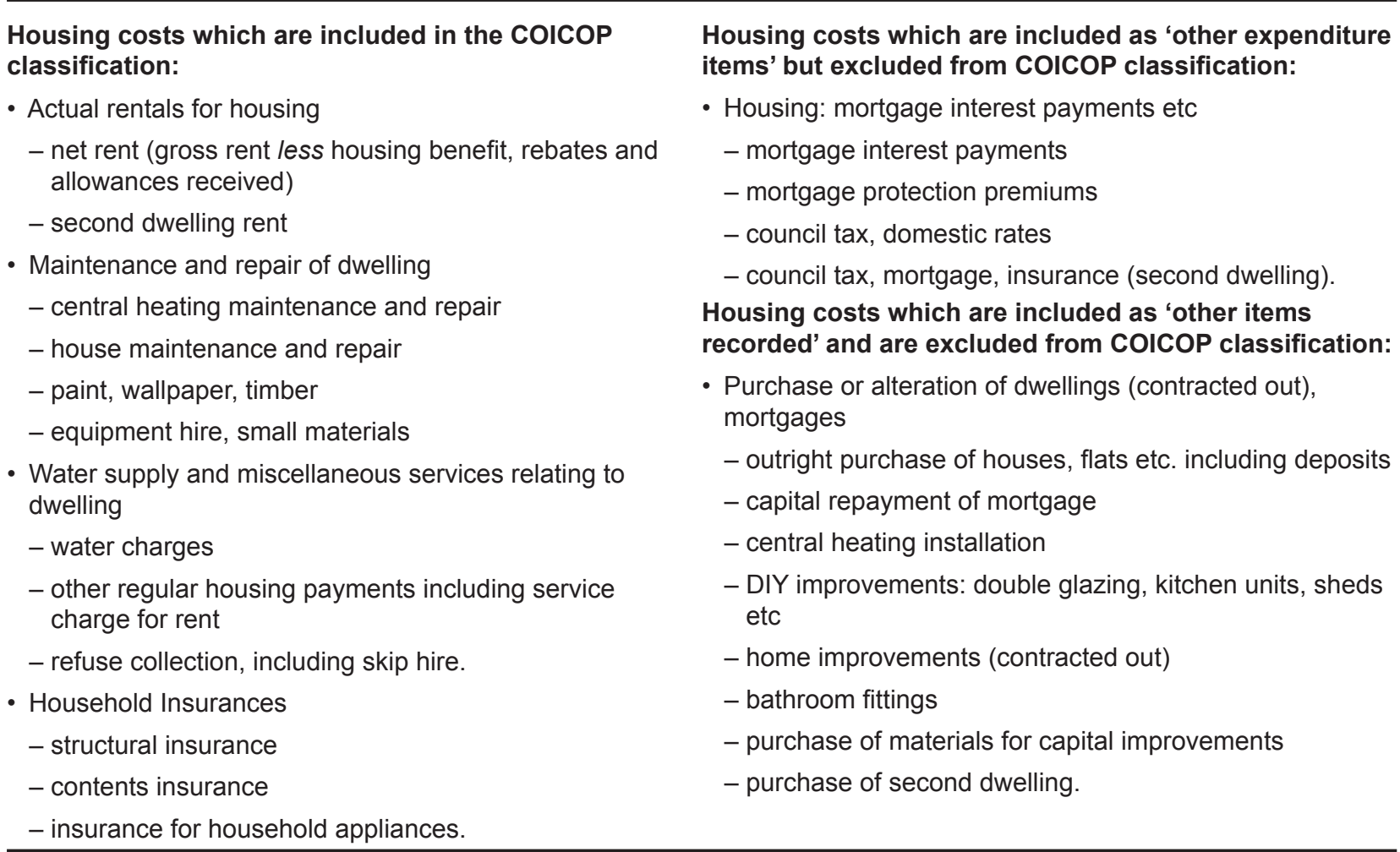

\section{Housing expenditure}

Table 2.2 shows expenditure on the items included in the comprehensive definition of housing expenditure. It also displays total household expenditure, which includes all expenditure items covered by the survey. The total expenditure figure reported here is therefore greater than the expenditure totals shown in the tables in Appendix $A$, as these exclude certain non-consumption costs.

Under the comprehensive definition of housing expenditure, UK households spent on average $£ 134.70$ a week on housing in 2010 , which equates to a fifth (20 per cent) of total weekly expenditure. The COICOP definition of housing expenditure (excluding fuel and power) on the other hand, gave an average of $£ 39.00$ per week for each household (see Table A1).

In 2010 spending was highest on mortgages (interest payments, protection premiums and capital repayments) at $£ 44.90$ a week. The next highest expenditure was on charges (council tax or domestic rates, water charges, refuse collection and other regular services) at $£ 27.20$ a week. This was followed by net rent at $£ 24.40$ a week and household alterations and improvements at $£ 19.70$ per week. Figure 2.1 provides a breakdown of housing expenditure items as a proportion of housing expenditure. 


\section{Figure 2.1 Housing expenditure items as a percentage of total housing expenditure, 2010 \\ United Kingdom}

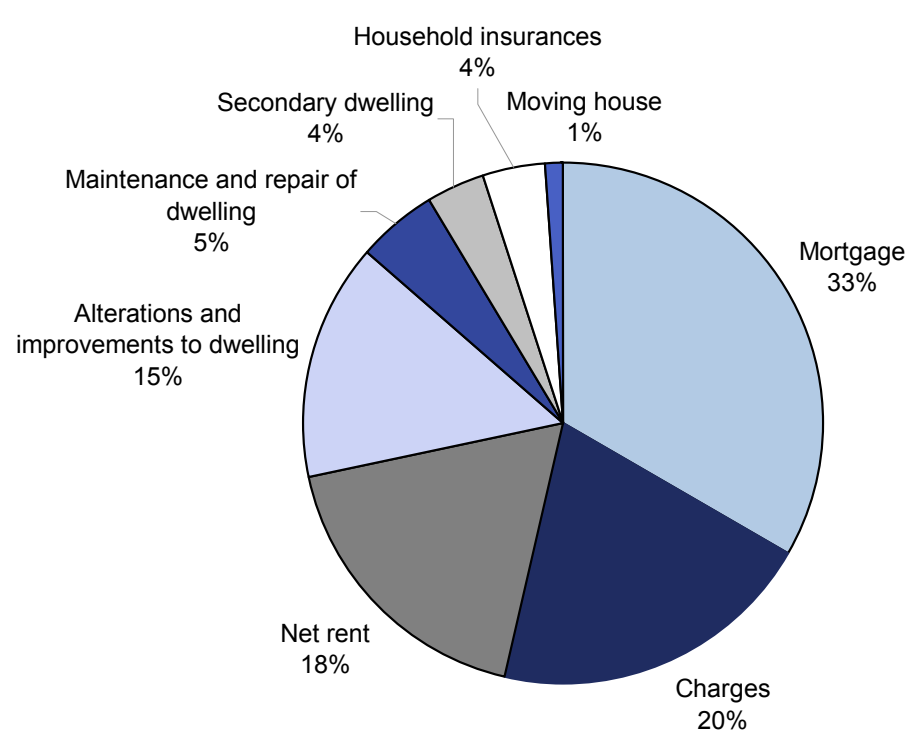

\section{Housing expenditure over time}

Overall, expenditure on housing has decreased over the last three years, from $£ 143.40$ in 2008 to $£ 134.70$ per week in 2010 . There was a decrease of $£ 4.10$ per week from 2008 to 2009 , which was followed by a further decrease of $£ 4.60$ between 2009 and 2010 . However, housing expenditure as a percentage of total expenditure has remained stable, decreasing by only 1 percentage point from 2008 (21 per cent) to 2010 (20 per cent). See Table 2.2 for a comparison of housing expenditure from 2008 to 2010.

Figure 2.2 presents the average weekly spend on each category of housing expenditure from 2008 to 2010. The largest decrease was seen in the mortgages category, which has decreased from $£ 57.20$ in 2008 to $£ 44.90$ in 2010 . Spending has remained relatively consistent for most other categories, with slight downward trends in alterations and improvements to dwellings and upward trends in charges such as council tax and water. 


\section{Figure 2.2 Housing expenditure 2008 to 2010 United Kingdom}

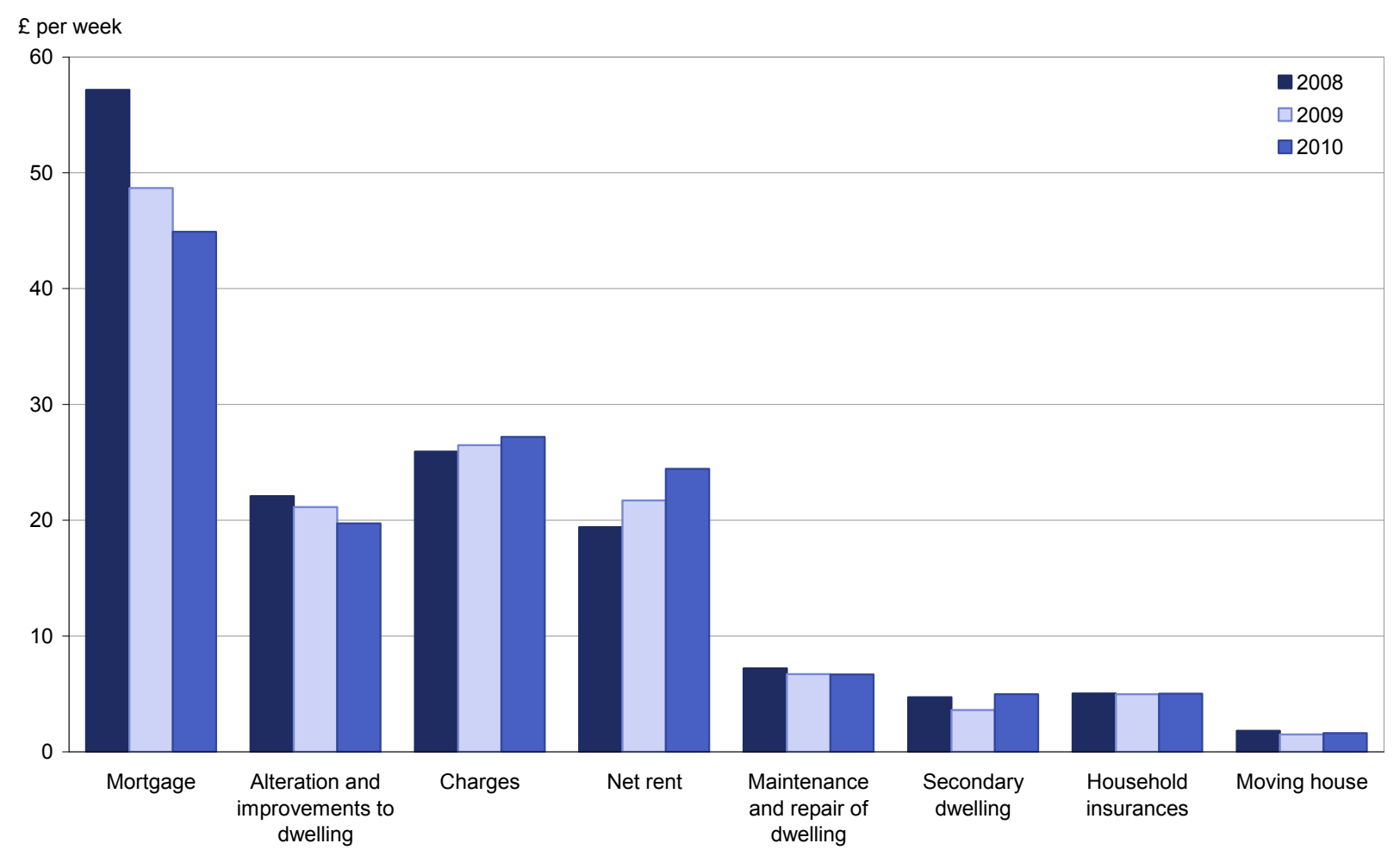

\section{Expenditure by gross income}

Table 2.3 presents housing expenditure by gross income decile group (a decile is one-tenth of the distribution). Overall, spending on housing increased with income. The highest income group spent $£ 318.20$ per week, more than twice the average for all income groups (£134.70) and more than six times that of the lowest income group (£49.30).

The categories that showed the greatest variation by income are mortgages, and alteration and improvements to dwellings. Figure 2.3 shows expenditure on mortgages to be consistently higher through income deciles, up to a weekly average of $£ 129.60$ in the highest income decile.

Expenditure on alteration and improvements to dwellings increased with income, and displayed a sharp increase in the tenth decile group to $£ 71.40$ per week, almost double that of the ninth decile group (£36.20). 


\section{Figure 2.3 Expenditure on selected items by gross income decile group, 2010 \\ United Kingdom}

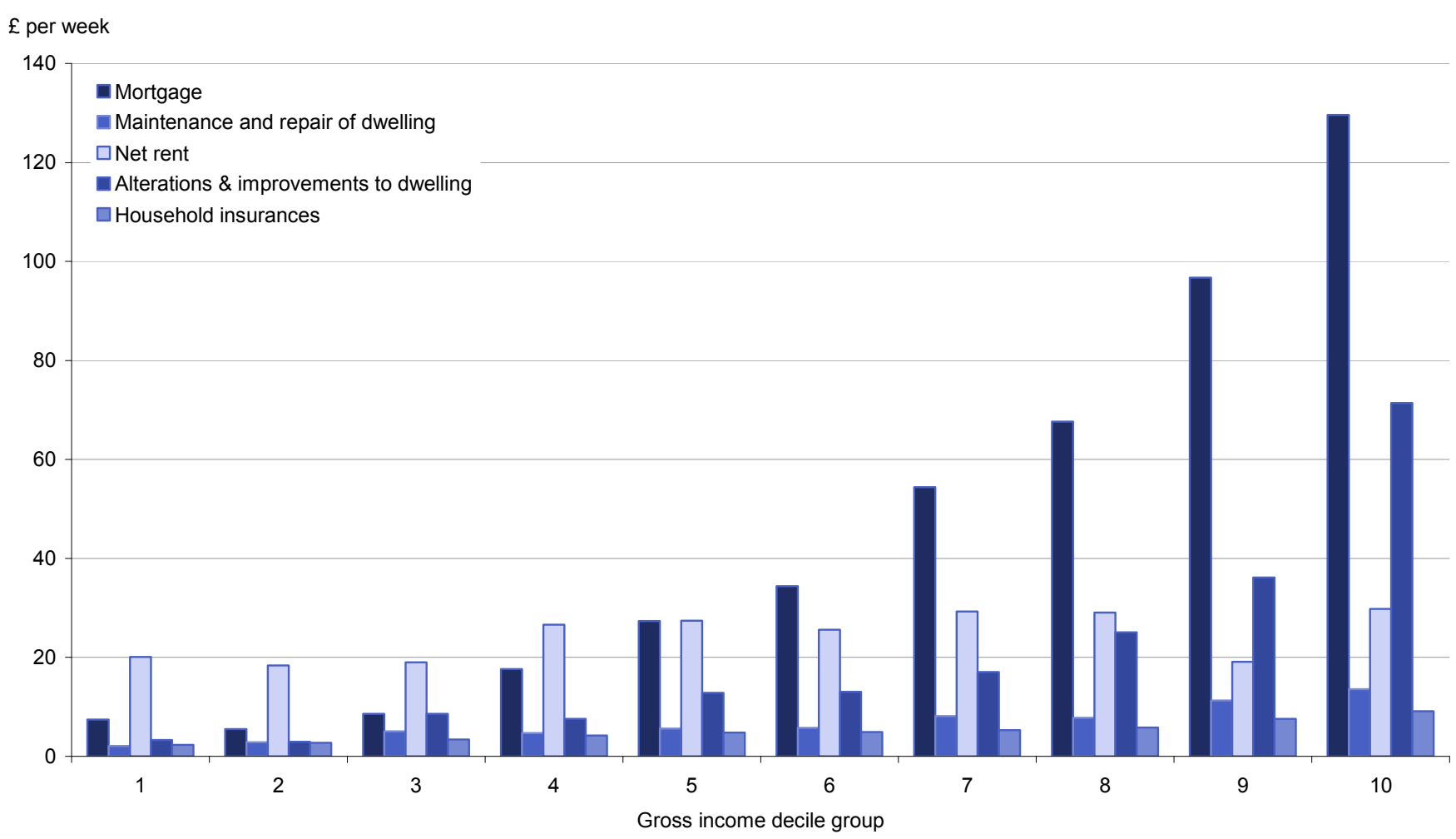

A different pattern can be seen when looking at net rent by income decile group; households in the second decile group spent the least on net rent at £18.30. Expenditure on rent was relatively consistent for higher income deciles, with the highest decile showing just the highest expenditure at $£ 29.80$. The exception was the ninth decile where expenditure was substantially lower at $£ 19.10$.

Expenditure on household insurances and on maintenance and repairs of dwellings increased slightly with income, but the increase was far less pronounced than for other categories.

\section{Expenditure by age of the household reference person}

Table 2.4 presents average weekly expenditure by age of household reference person (HRP, defined in Appendix B). Figure 2.4 presents spending on three key housing expenditure categories by age of HRP. Average weekly expenditure on mortgages peaked at $£ 79.70$ for households with an HRP aged 30 to 49. Average weekly expenditure for households with an HRP within the age range 50 to 64 was $£ 42.20$, and households with an HRP under 30 was even lower at $£ 35.30$. However, the average weekly spend for household alterations and improvements was highest for households with an HRP aged 50 to 64 at £26.20. Net rent expenditure decreased as the age of the HRP increased. Average weekly expenditure for households with an HRP under the age of 30 was $£ 65.90$, compared with £31.90 for households with an HRP aged between 30 and 49, and $£ 7.00$ for households with an HRP aged over 75 . 
Figure 2.4 Expenditure on selected items by age of household reference person, 2010

United Kingdom

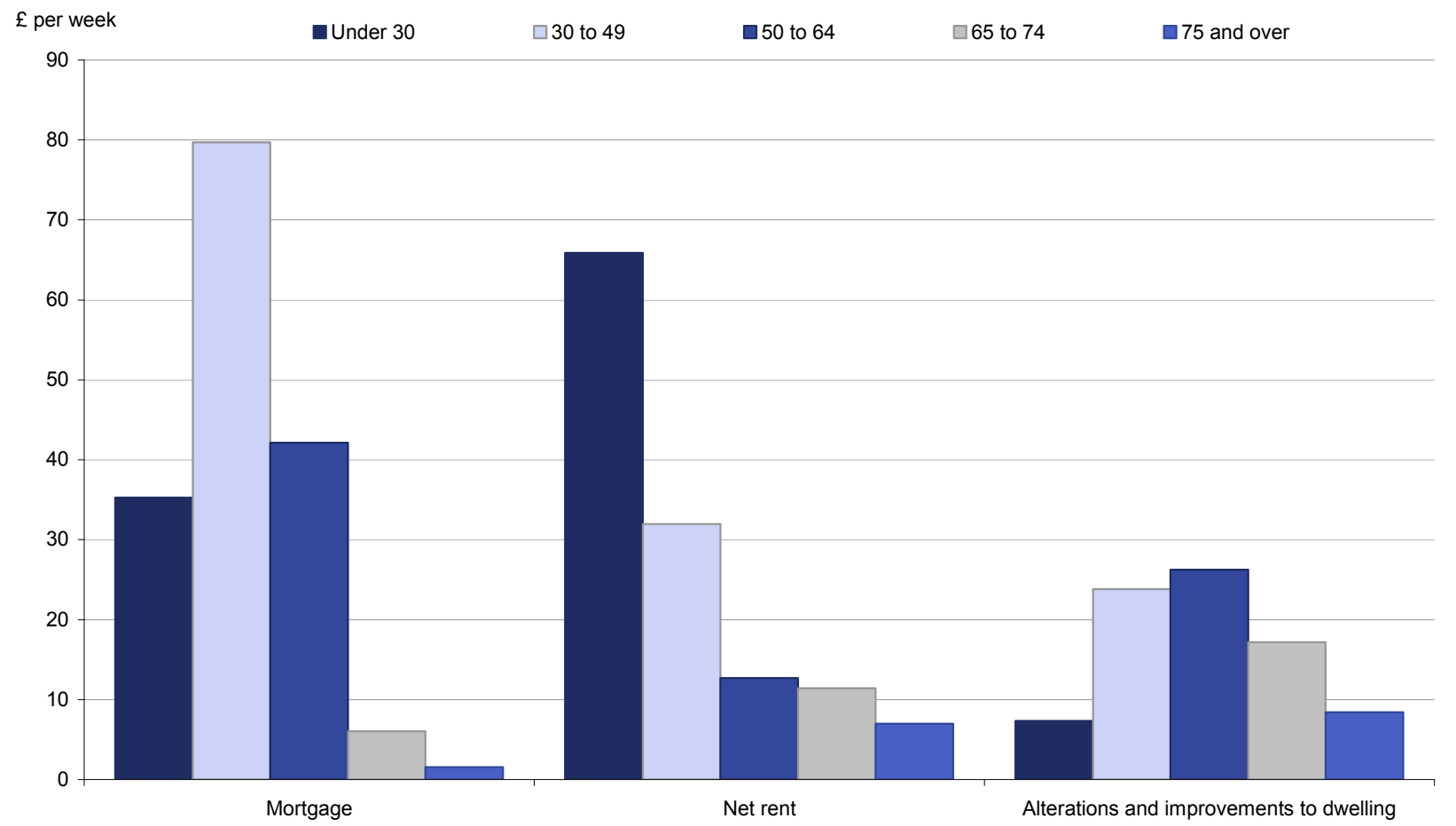

Figure 2.5 shows that expenditure on net rent for households with an HRP aged under 30 has decreased from $£ 72.00$ in 2009 to $£ 65.90$ in 2010 . There were slight increases for HRP age groups over 30 and below 75 .

Figure 2.5 Expenditure on net rent by age of household reference person, 2009 and 2010

United Kingdom

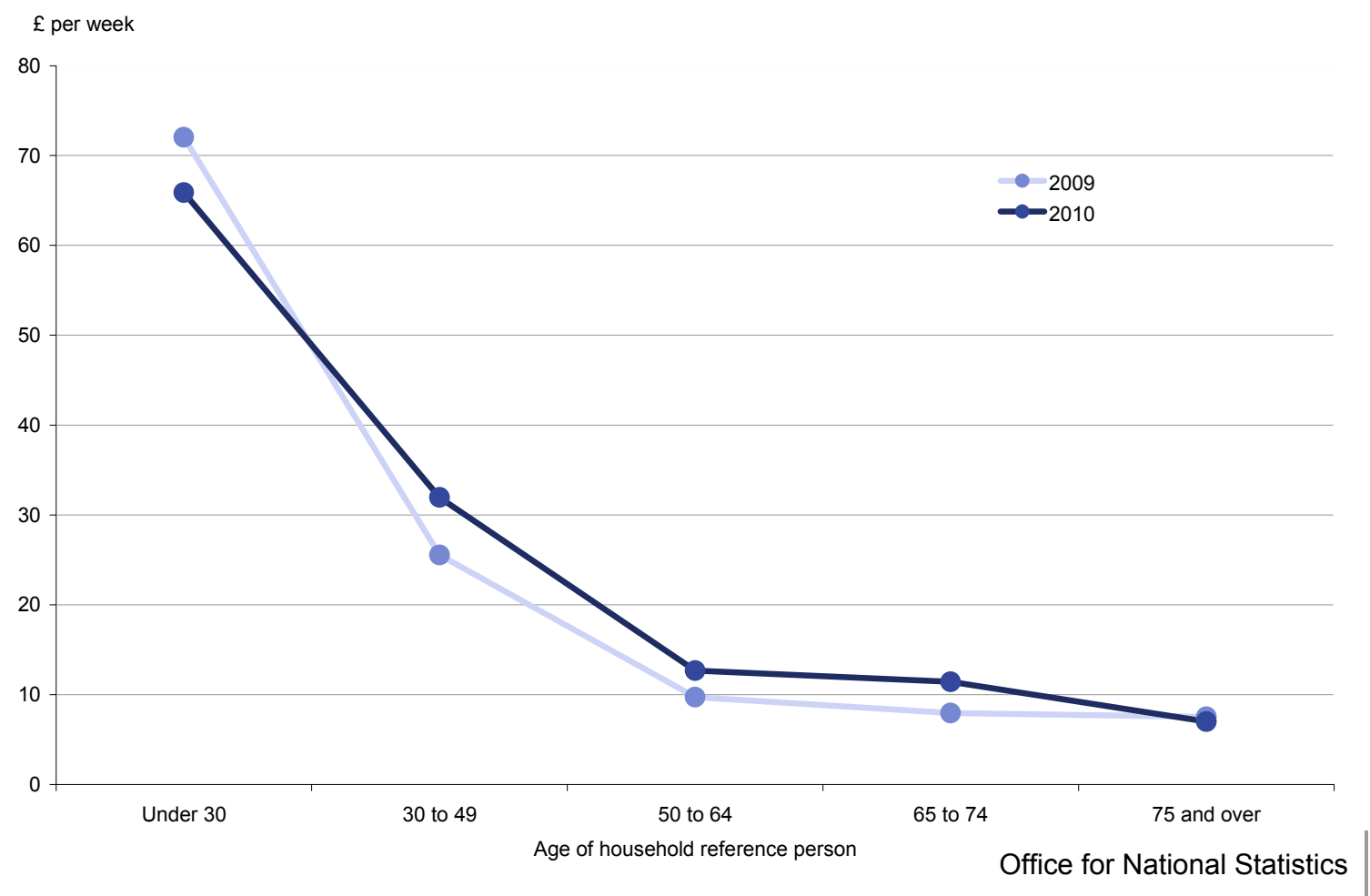




\section{Expenditure by region}

Table 2.5, Figure 2.6, and Figure 2.7 show housing expenditure by UK country and region. Looking first at expenditure by country (Figure 2.6), households in England spent the most on housing at $£ 139.20$ a week, followed by Scotland (£121.80), Wales (£112.80) and Northern Ireland (£80.10).

\section{Figure 2.6 Housing expenditure by Country, 2010 United Kingdom}

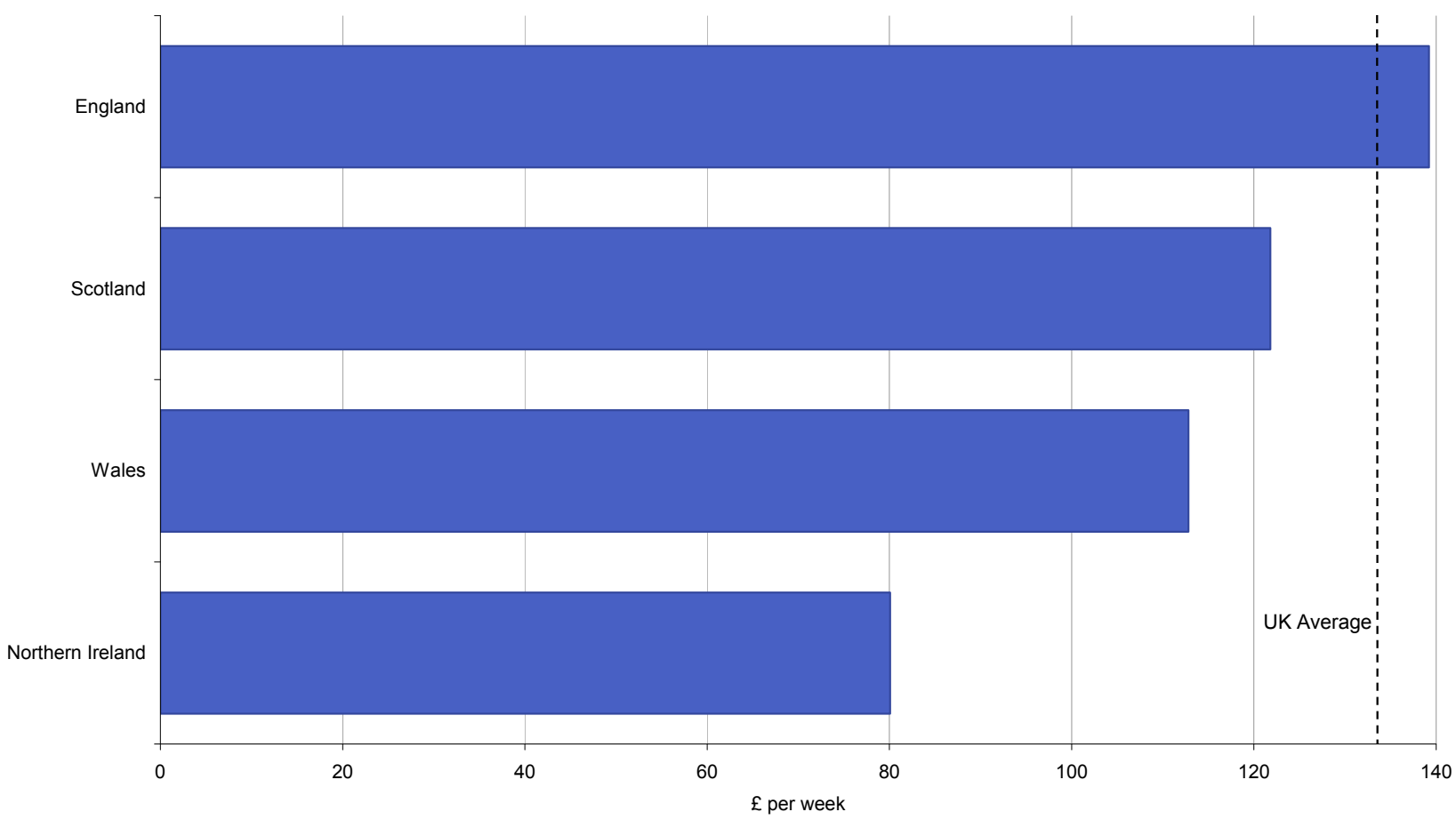

Figure 2.7 shows housing expenditure by region and country (excluding England). Five English regions had average weekly household spends that were greater than the UK average. Housing expenditure was greatest in London at $£ 199.00$ a week, followed by the South East (£155.70) and South West (£153.10). Expenditure was lowest in Northern Ireland with an average spend of $£ 80.10$. The lowest spending in England was in the North East, where average weekly housing costs were $£ 98.00$. 


\section{Figure 2.7 Housing expenditure by UK regions and Countries, 2010}

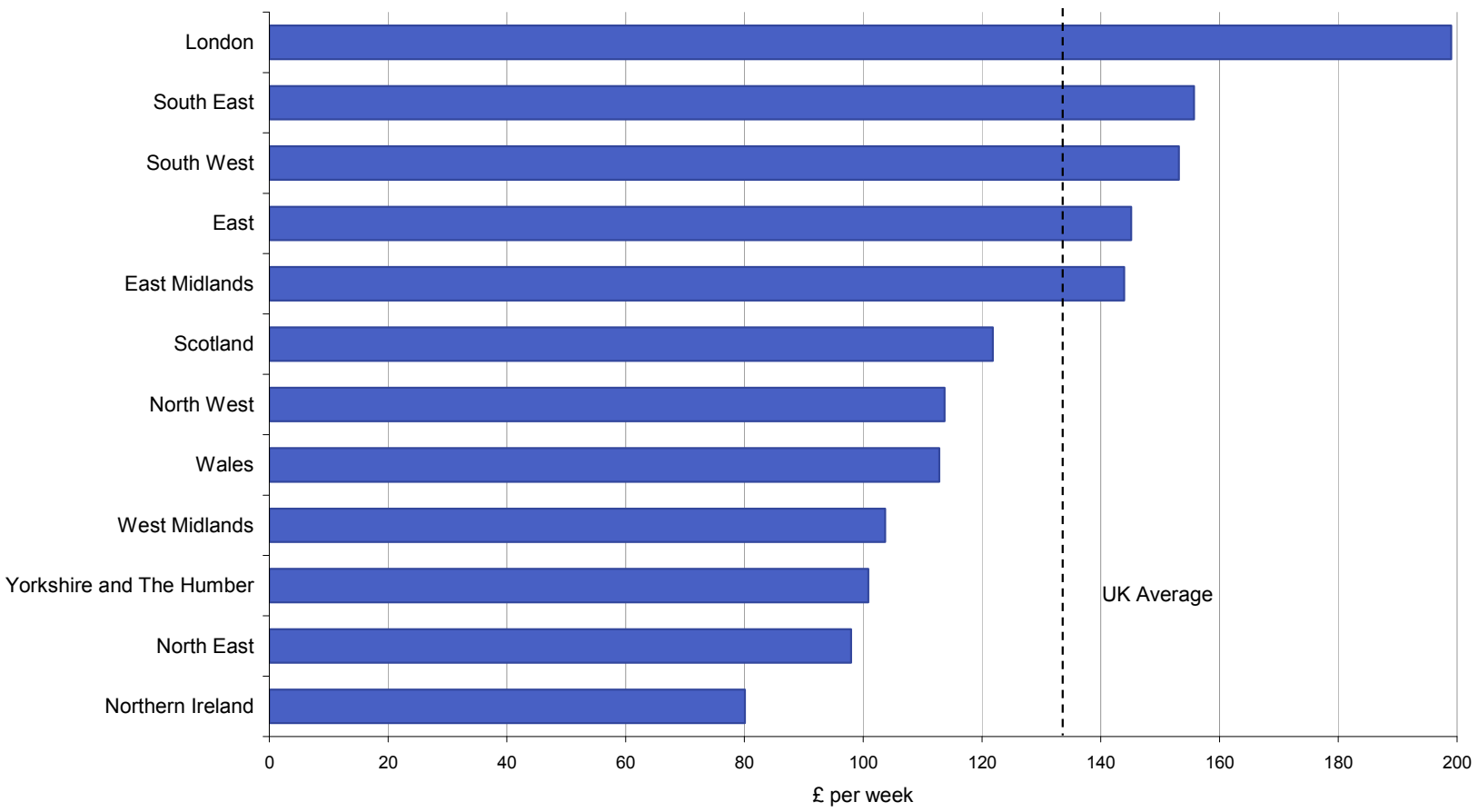

Variations in the total spending on housing are largely due to differences between regions in the average amount spent on rent and mortgages. Table 2.5 presents housing expenditure by country and region, while Figures 2.8 and 2.9 present the percentage difference in each region from the UK average for net rent and mortgages, respectively. It is important to note that these figures include all households. Average expenditure on rent only by renters, and mortgages only by mortgage holders is examined later in the chapter.

Average weekly expenditure on net rent was $£ 24.40$ (Table 2.5). Figure 2.8 shows that expenditure was more than twice the average in London ( 54.60$)$, and slightly above average in the South East (£25.50); these were the only regions to exceed the UK average. Spending on net rent was lowest in Northern Ireland with an average weekly expenditure of $£ 13.10$, closely followed by the North East of England where the average weekly net rent was $£ 15.80$ 
Figure 2.8 Percentage difference compared with UK average for net rent by UK Countries and regions, 2010

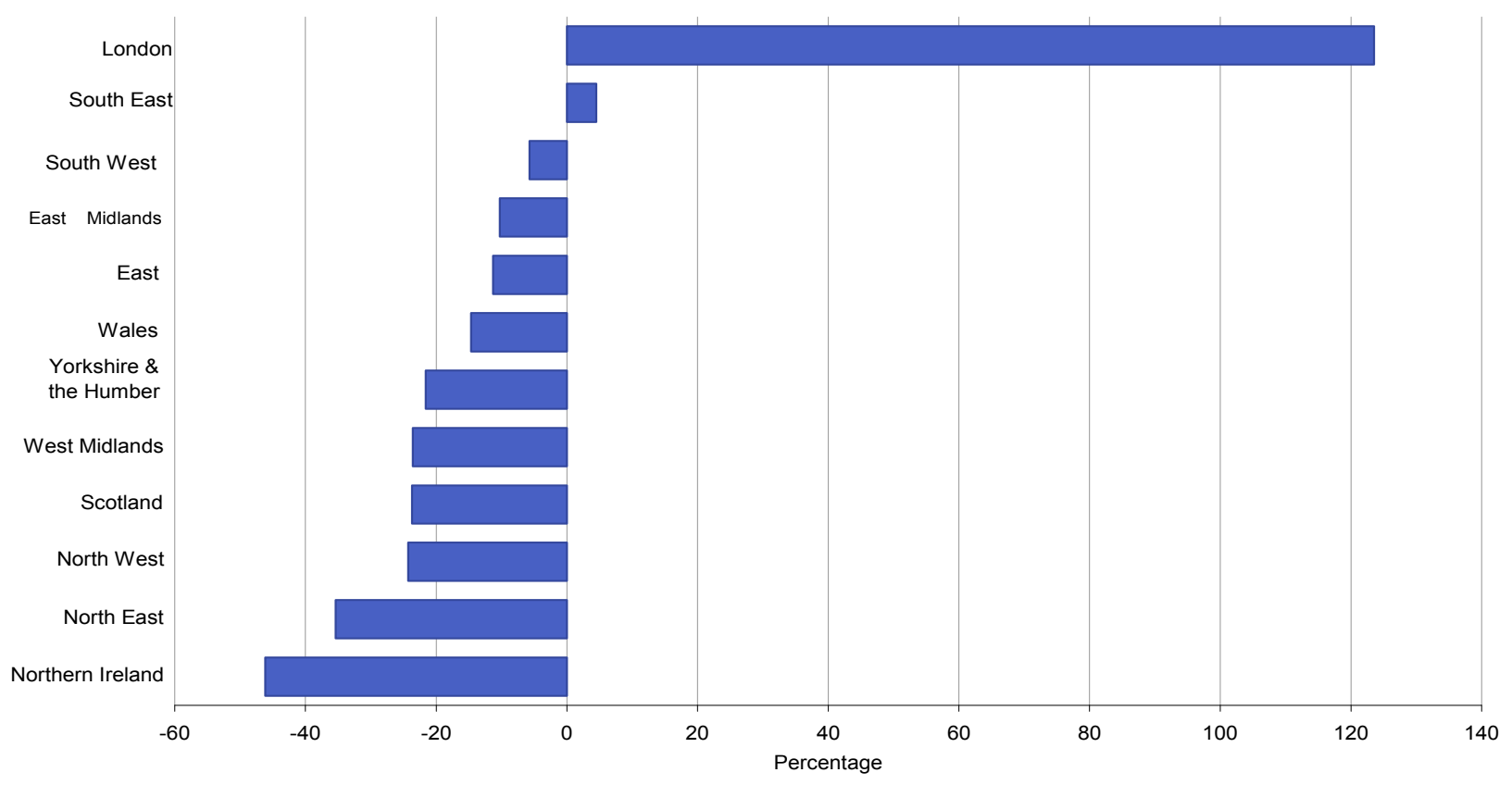

Figure 2.9 shows that London, the South East and the East of England had average mortgage payments above the UK average of $£ 44.90$; all other regions spent less on mortgages than the UK average. Expenditure on mortgages was lowest in Wales with average weekly mortgage payments of $£ 34.90$.

Figure 2.9 Percentage difference compared with UK average for mortgage payments by UK Countries and regions, 2010

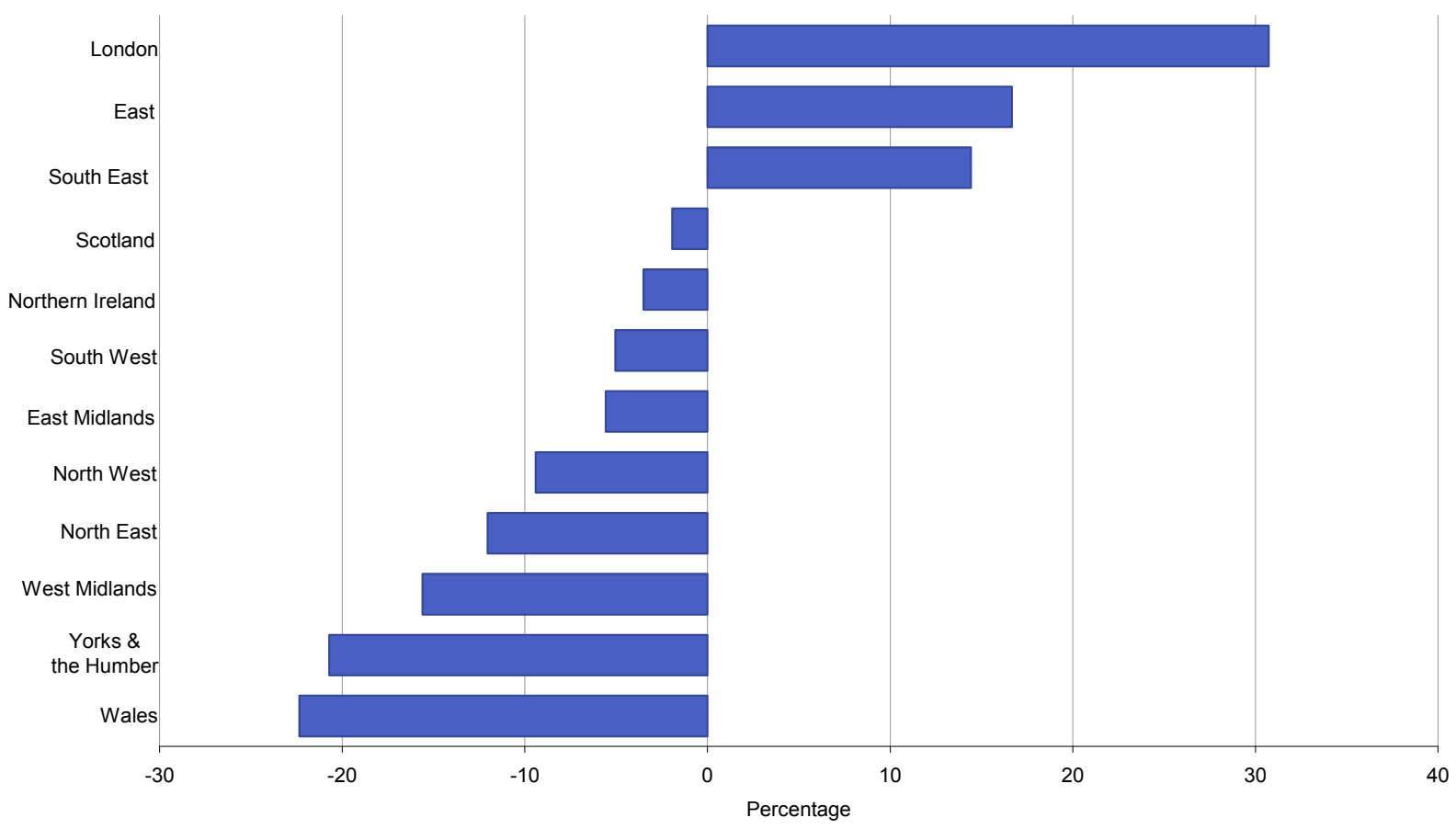




\section{Expenditure by socio-economic classification}

Figure 2.10 and Table 2.6 present housing expenditure by socio-economic classification of the household reference person (HRP). Households with an HRP in the 'large employer and higher managerial' occupation group spent the most, at $£ 309.30$ per week: more than twice that of households with an HRP in the 'routine' occupation group, where the average weekly spend was $£ 114.30$.

\section{Figure 2.10 Housing expenditure by socio-economic classification of household reference person, 2010 United Kingdom}

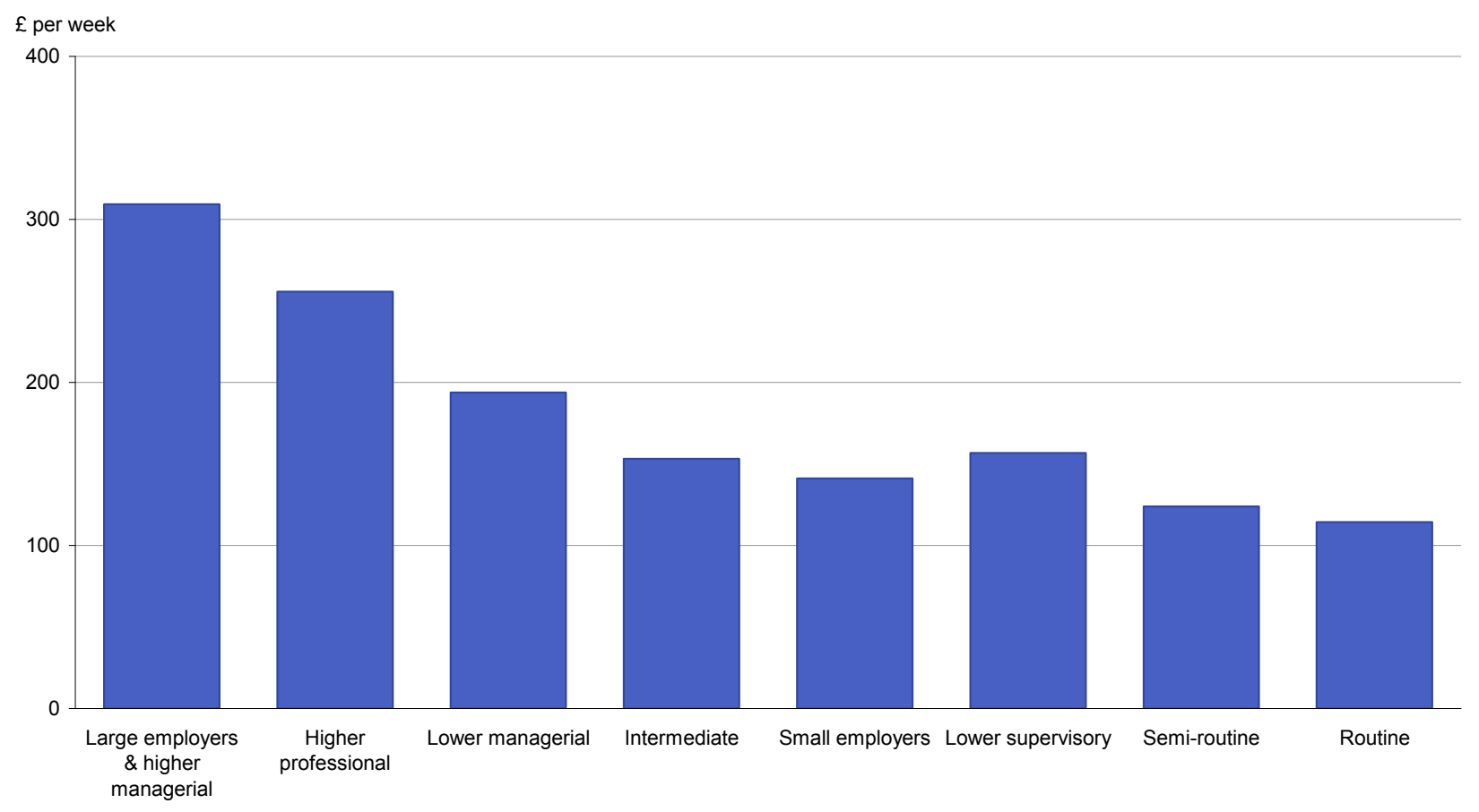

Figure 2.11 presents expenditure on selected items by the socio-economic classification of the HRP. Overall, expenditure followed a similar pattern as described above, with those with an HRP classified as 'large employer and higher managerial' spending more than those in 'routine' occupations. The exception to this was net rent where the opposite pattern is observed and the highest expenditure was for the 'routine' occupation group, reflecting the high number of renters in this group. 


\section{Figure 2.11 Expenditure on selected items by socio-economic classification of household reference person, 2010 United Kingdom}

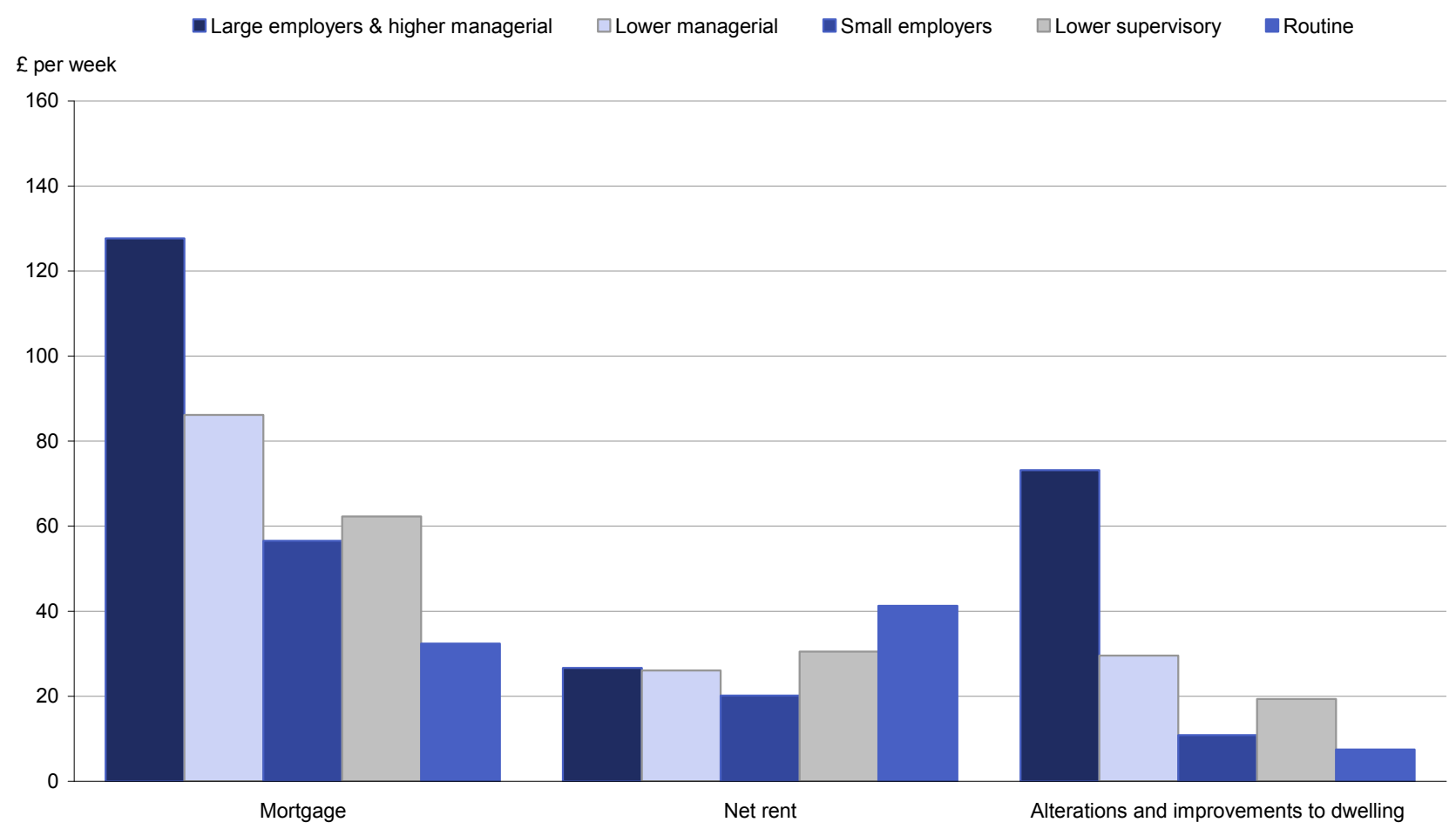

\section{Analysis of housing costs for renters and mortgage holders}

The following analysis looks at average expenditure on net rent for households that report spending on net rent and average expenditure on mortgages for mortgage holders. This is the only place in Family Spending where averages are not across all households. Excluding households with nil expenditure for net rent and mortgages provides a more informative picture of expenditure on these items.

Table 2.8 provides expenditure on rent over the last three years. In 2010 renters spent on average $£ 74.40$ per week on net rent. Table 2.9 provides expenditure on mortgages over the last three years. In 2010 the average weekly expenditure on mortgages by mortgage holders was $£ 130.80$.

Table 2.10 and Figure 2.12 present average weekly expenditure for the relevant households by income decile group for mortgage holders and renters.

Figure 2.12 shows a steady increase in net rent as the income decile increases. Households in the first income decile spent $£ 31.70$ on net rent, compared with $£ 146.80$ in the ninth income decile and $£ 255.20$ in the tenth income decile. It should be noted, however, that a relatively small number of households in the highest income group paid rent. The estimate of net costs for this income group should therefore be viewed with caution. Average expenditure for mortgages followed a roughly similar pattern to net rent, increasing towards the higher income deciles. The fluctuations in the lower decile groups may be due to a low number of mortgage holders in this decile group, and should be viewed with caution. 
Figure 2.12 Expenditure on net rent by renters, and mortgages by mortgage holders, by gross income decile group, 2010 United Kingdom

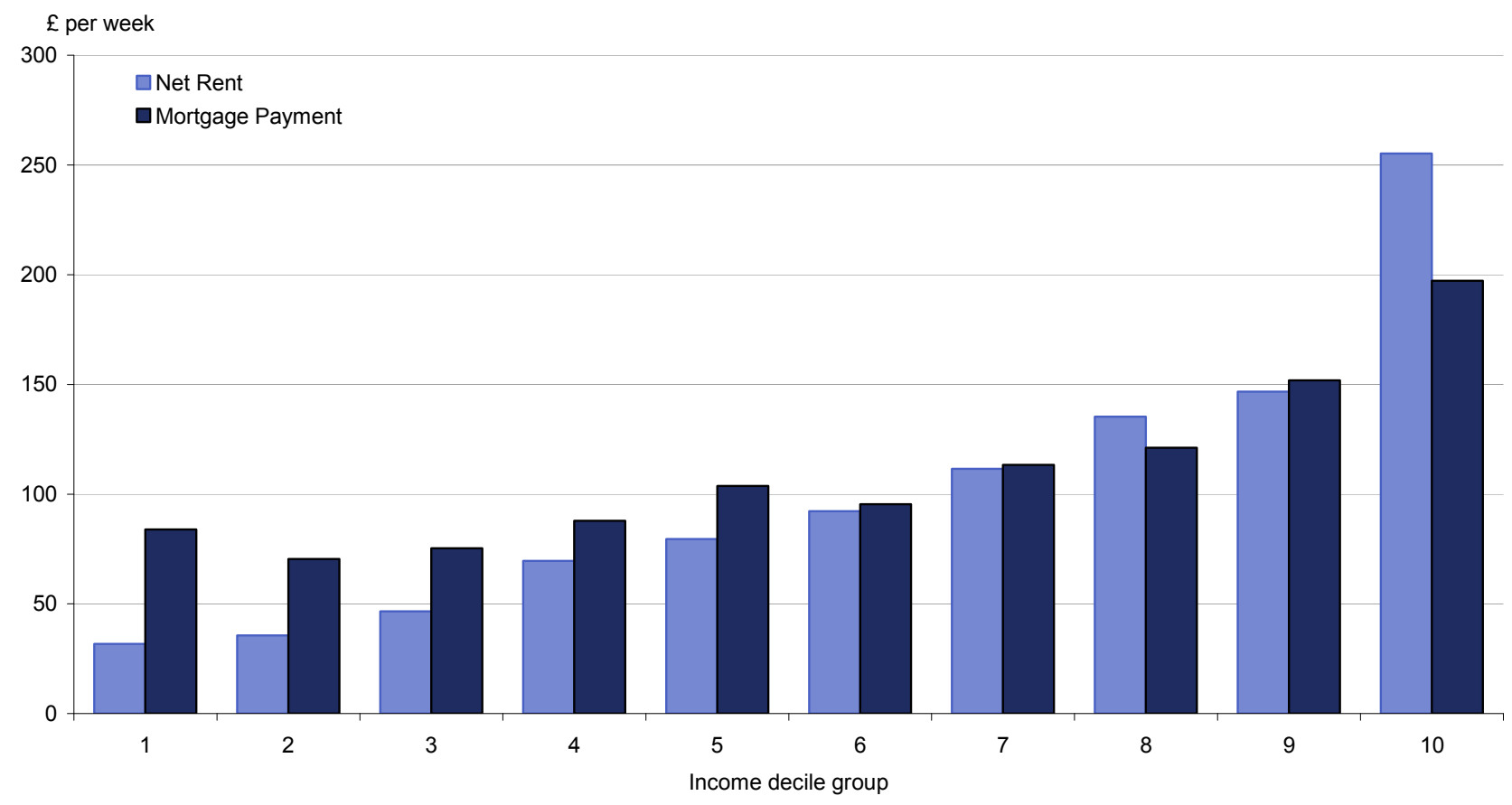

Figure 2.13, and Figure 2.14 show net rent and mortgage expenditure, respectively, averaged across renters and mortgage holders, by country and region. The figures are presented in Table 2.11 .

\section{Figure 2.13 Expenditure on net rent by renters, by UK Countries and regions, 2010}

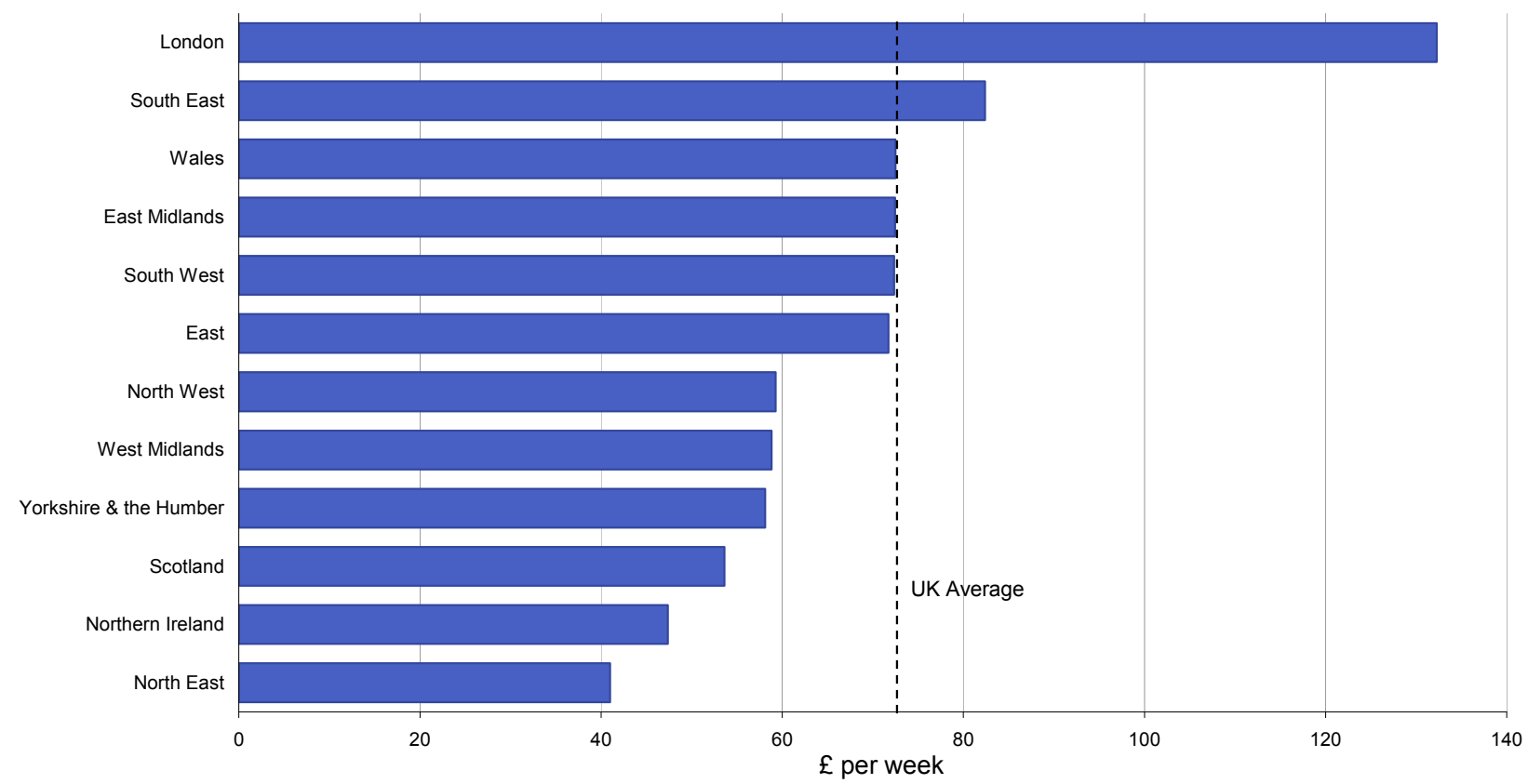




\section{Figure 2.14 Expenditure on mortgages by mortgage holders by UK Countries and regions, 2010}

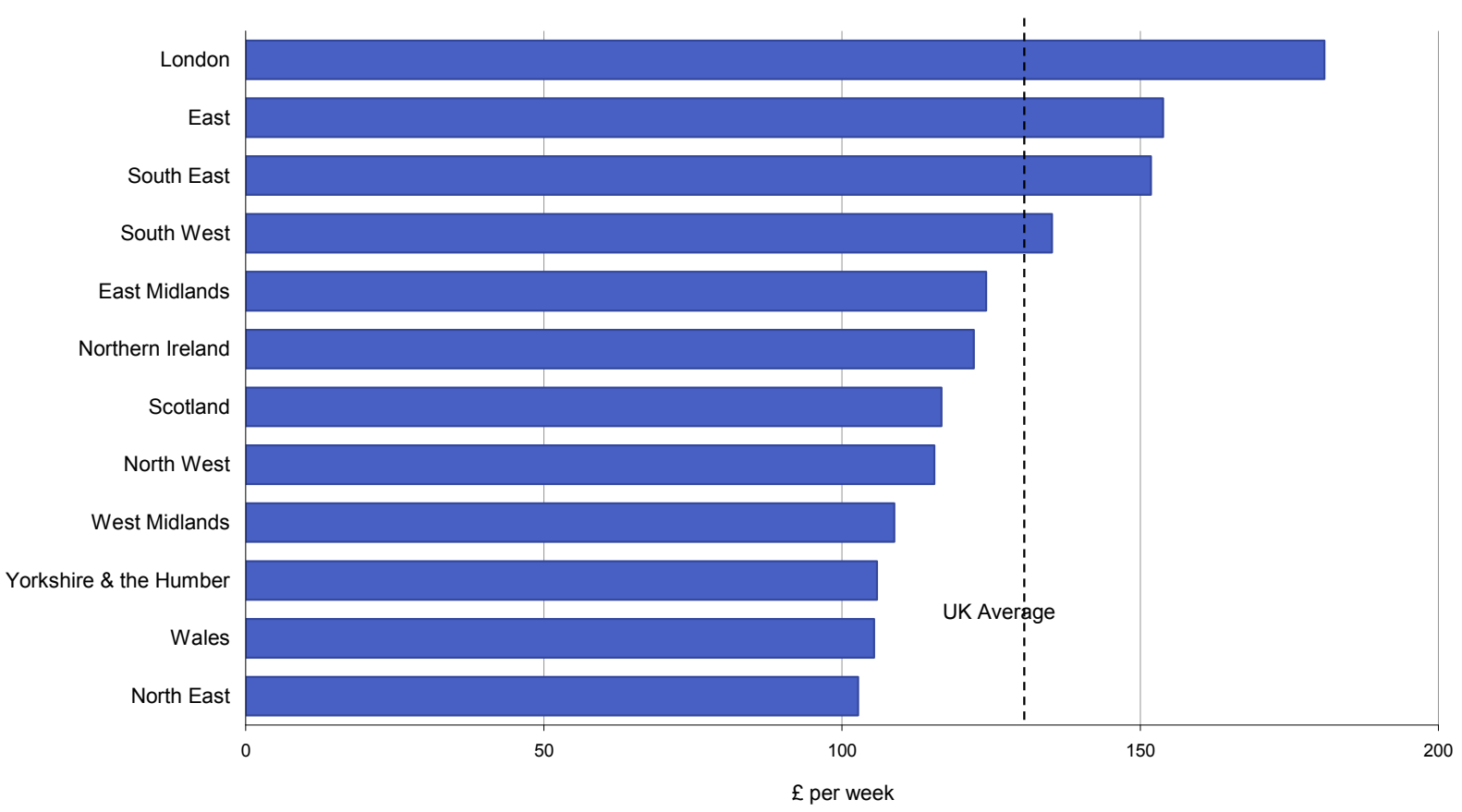

Table 2.11 shows the country with the highest average spend on net rent was England, which at $£ 77.50$ a week was above the UK average of $£ 74.40$. Wales had the next highest average weekly expenditure among UK countries on net rent at $£ 72.50$, followed by Scotland (£53.60) and Northern Ireland with the lowest average weekly spend (£47.40).

There were two regions that had average weekly expenditures on net rent greater than the UK average of $£ 74.40$. London spent the most on net rent by a substantial margin ( 1132.30 ), followed by the South East (£82.40). All the other regions had average weekly spends lower than the UK average. The region with the lowest average weekly expenditure on net rent was North East (£41.00).

Looking at expenditure on mortgages by country (Table 2.11), England at $£ 134.20$ was the only country that had an average weekly expenditure on mortgages greater than the UK average of $£ 130.80$ per week. Closely following England was Northern Ireland (£122.10), Scotland (£116.70) and finally Wales (£105.40). The differences between average weekly expenditure by country are much smaller for mortgages compared with net rent.

Four regions had a greater average weekly spend on mortgage payments than the UK average (Figure 2.14). London had the highest average spend at $£ 180.90$ per week, although the difference was less pronounced than for net rent. The next highest regions were the East (£153.80), the South East (£151.80) and the South West (£135.20). The region with the lowest weekly spend was North East at $£ 102.70$ per week; just under $£ 30$ a week lower than the UK average. 
Table 2.2 Housing expenditure, 2008 to 2010 United Kingdom

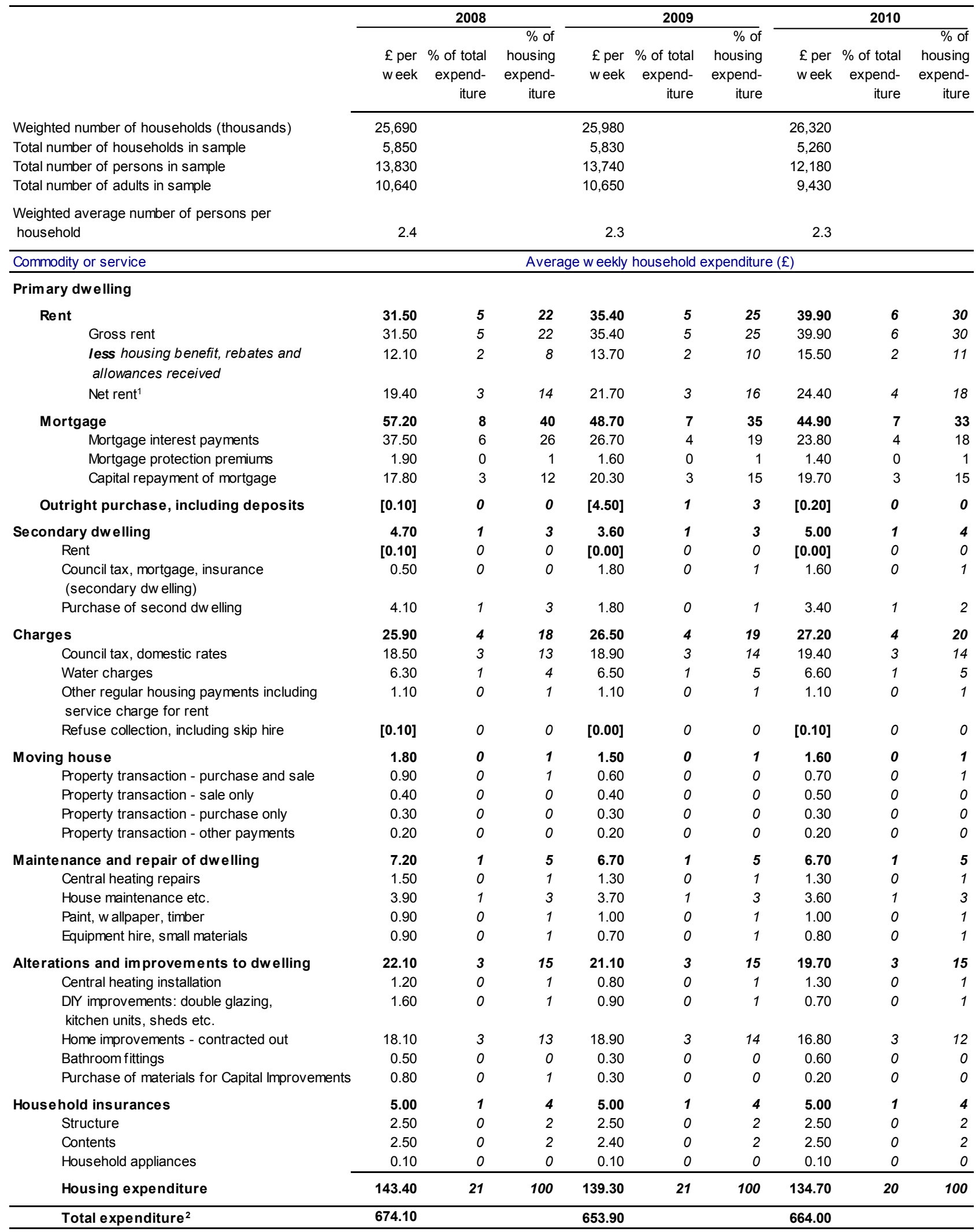

Note: Please see page xiii for symbols and conventions used in this report.

1 The figure included in total expenditure is net rent as opposed to gross rent.

2 This total includes all categories recorded in the LCF, including those outside the 'COICOP' total expenditure 
Table 2.3 Housing expenditure by gross income decile group, 2010 United Kingdom

\begin{tabular}{|c|c|c|c|c|c|c|c|c|c|c|c|}
\hline & \multicolumn{11}{|c|}{ Gross income decile group } \\
\hline & 1 & 2 & 3 & 4 & 5 & 6 & 7 & 8 & 9 & 10 & All \\
\hline Weighted number of households (thousands) & 2,630 & 2,640 & 2,630 & 2,630 & 2,630 & 2,640 & 2,630 & 2,640 & 2,630 & 2,630 & 26,320 \\
\hline Total number of households in sample & 510 & 530 & 540 & 550 & 550 & 530 & 530 & 520 & 510 & 500 & 5,260 \\
\hline Total number of persons in sample & 690 & 860 & 960 & 1,150 & 1,280 & 1,290 & 1,410 & 1,430 & 1,530 & 1,580 & 12,180 \\
\hline Total number of adults in sample & 570 & 670 & 780 & 900 & 970 & 1,010 & 1,090 & 1,090 & 1,170 & 1,170 & 9,430 \\
\hline $\begin{array}{l}\text { Weighted average number of persons per } \\
\text { household }\end{array}$ & 1.3 & 1.6 & 1.8 & 2.1 & 2.4 & 2.4 & 2.7 & 2.8 & 3.0 & 3.2 & 2.3 \\
\hline Commodity or service & \multicolumn{11}{|c|}{ Average w eekly household expenditure $(£)$} \\
\hline \multicolumn{12}{|l|}{ Prim ary dw elling } \\
\hline Rent & 71.80 & 58.10 & 46.80 & 43.70 & 38.80 & 28.90 & 31.70 & 29.50 & 19.80 & 29.80 & 39.90 \\
\hline Gross rent & 71.80 & 58.10 & 46.80 & 43.70 & 38.80 & 28.90 & 31.70 & 29.50 & 19.80 & 29.80 & 39.90 \\
\hline $\begin{array}{l}\text { less housing benefit, rebates and } \\
\text { allowances received }\end{array}$ & 51.80 & 39.70 & 27.80 & 17.00 & 11.40 & 3.30 & 2.50 & [0.50] & {$[0.70]$} & [0.00] & 15.50 \\
\hline Net rent ${ }^{1}$ & 20.10 & 18.30 & 19.00 & 26.60 & 27.40 & 25.60 & 29.30 & 29.00 & 19.10 & 29.80 & 24.40 \\
\hline Mortgage & 7.40 & 5.50 & 8.60 & 17.60 & 27.40 & 34.40 & 54.40 & 67.60 & 96.70 & 129.60 & 44.90 \\
\hline Mortgage interest payments & 5.00 & 2.80 & 4.50 & 10.20 & 12.80 & 18.20 & 28.40 & 35.00 & 51.60 & 69.20 & 23.80 \\
\hline Mortgage protection premiums & [0.20] & [0.10] & 0.40 & 0.40 & 0.80 & 1.40 & 1.60 & 2.60 & 3.20 & 3.40 & 1.40 \\
\hline Capital repayment of mortgage & 2.20 & 2.50 & 3.70 & 7.00 & 13.80 & 14.80 & 24.40 & 30.10 & 41.90 & 56.90 & 19.70 \\
\hline Outright purchase, including deposits & - & {$[0.10]$} & {$[0.20]$} & - & {$[0.00]$} & [0.10] & - & {$[0.20]$} & {$[0.80]$} & [0.40] & [0.20] \\
\hline Secondary dwelling & [0.30] & {$[0.10]$} & {$[0.50]$} & [0.40] & [1.50] & {$[1.20]$} & [1.90] & {$[6.00]$} & 19.20 & 18.70 & 5.00 \\
\hline Rent & & {$[0.00]$} & & {$[0.00]$} & {$[0.10]$} & - & {$[0.10]$} & & - & [0.20] & {$[0.00]$} \\
\hline $\begin{array}{l}\text { Council tax, mortgage, insurance } \\
\text { (secondary dw elling) }\end{array}$ & [0.30] & - & {$[0.30]$} & {$[0.40]$} & [1.30] & [1.10] & {$[1.30]$} & {$[1.60]$} & [5.00] & 4.30 & 1.60 \\
\hline Purchase of second dw elling & - & [0.10] & {$[0.10]$} & - & {$[0.10]$} & {$[0.10]$} & {$[0.50]$} & [4.00] & [14.00] & {$[14.20]$} & 3.40 \\
\hline Charges & 13.30 & 16.50 & 21.30 & 25.00 & 27.10 & 29.10 & 30.80 & 32.80 & 34.70 & 41.50 & 27.20 \\
\hline Council tax, domestic rates & 6.60 & 9.60 & 13.80 & 17.20 & 19.80 & 21.60 & 22.80 & 24.20 & 26.40 & 31.80 & 19.40 \\
\hline Water charges & 5.50 & 5.70 & 5.70 & 6.40 & 6.30 & 6.90 & 7.00 & 7.00 & 7.40 & 8.40 & 6.60 \\
\hline \multicolumn{12}{|l|}{ Other regular housing payments } \\
\hline including service charge for rent & 1.30 & 1.20 & 1.80 & 1.40 & 1.00 & 0.60 & 0.80 & 1.40 & 0.70 & 1.10 & 1.10 \\
\hline Refuse collection, including skip hire & - & - & - & {$[0.00]$} & {$[0.00]$} & - & [0.30] & {$[0.20]$} & {$[0.20]$} & [0.10] & [0.10] \\
\hline $\begin{array}{l}\text { Moving house } \\
\quad \text { Property transaction - purchase and }\end{array}$ & \multicolumn{10}{|c|}{ Property transaction - purchase and } & 1.60 \\
\hline sale & {$[0.00]$} & {$[0.00]$} & [0.50] & {$[0.50]$} & [1.00] & {$[0.60]$} & [0.50] & {$[0.60]$} & [1.30] & [1.60] & 0.70 \\
\hline Property transaction - sale only & - & {$[0.50]$} & {$[0.20]$} & {$[0.70]$} & {$[0.00]$} & - & {$[0.30]$} & {$[0.50]$} & {$[0.70]$} & [1.70] & 0.50 \\
\hline Property transaction - purchase only & - & & [0.20] & {$[0.10]$} & {$[0.20]$} & [0.30] & {$[0.20]$} & [0.30] & [0.90] & [0.60] & 0.30 \\
\hline Property transaction - other payments & - & [0.10] & {$[0.10]$} & {$[0.10]$} & {$[0.20]$} & {$[0.10]$} & {$[0.20]$} & {$[0.20]$} & {$[0.40]$} & [0.40] & 0.20 \\
\hline Maintenance and repair of dwelling & 2.10 & 2.80 & 5.10 & 4.70 & 5.60 & 5.80 & 8.20 & 7.80 & 11.30 & 13.60 & 6.70 \\
\hline Central & 0.50 & 0.70 & 0.70 & 1.10 & 1.10 & 1.40 & 1.70 & 1.90 & 1.90 & 2.20 & 1.30 \\
\hline House maintenance etc. & 0.80 & 1.50 & 3.20 & 2.70 & 2.50 & 2.80 & 3.50 & 3.80 & 6.40 & 8.50 & 3.60 \\
\hline Paint, w allpaper, timber & 0.60 & 0.40 & 0.80 & 0.60 & 0.80 & 0.80 & 2.10 & 0.90 & 1.70 & 1.70 & 1.00 \\
\hline Equipment hire, small materials & 0.20 & 0.20 & 0.40 & 0.30 & 1.20 & 0.80 & 0.80 & 1.20 & 1.30 & 1.20 & 0.80 \\
\hline Alterations and improvements to dwelling & 3.30 & 2.90 & 8.60 & 7.60 & 12.80 & 13.00 & 17.10 & 25.10 & 36.20 & 71.40 & 19.80 \\
\hline Central heating installation & [0.70] & [0.90] & {$[0.60]$} & [1.70] & [1.40] & {$[0.80]$} & [1.10] & [1.70] & [1.50] & 3.30 & 1.40 \\
\hline $\begin{array}{l}\text { DIY improvements: double glazing, } \\
\text { kitchen units, sheds etc. }\end{array}$ & \multicolumn{10}{|c|}{ kitchen units, sheds etc. } & 0.70 \\
\hline Home improvements - contracted out & 2.40 & 1.70 & 7.80 & 4.80 & 10.00 & 9.60 & 14.20 & 21.90 & 29.70 & 66.10 & 16.80 \\
\hline Bathroom fittings & [0.10] & [0.20] & {$[0.00]$} & {$[0.00]$} & {$[0.60]$} & [2.50] & {$[0.10]$} & [0.40] & [1.70] & [0.70] & 0.60 \\
\hline \multicolumn{7}{|l|}{ Purchase of materials for capital } & [0.40] & [0.50] & {$[0.10]$} & [0.30] & 0.20 \\
\hline Household insurances & 2.30 & 2.70 & 3.40 & 4.20 & 4.80 & 4.90 & 5.30 & 5.80 & 7.60 & 9.10 & 5.00 \\
\hline Structure & 1.00 & 1.30 & 1.60 & 1.90 & 2.40 & 2.40 & 2.60 & 2.90 & 3.80 & 4.70 & 2.50 \\
\hline Contents & 1.20 & 1.40 & 1.70 & 2.10 & 2.40 & 2.40 & 2.60 & 2.80 & 3.60 & 4.30 & 2.50 \\
\hline Household appliances & {$[0.00]$} & {$[0.00]$} & {$[0.10]$} & {$[0.10]$} & {$[0.10]$} & {$[0.10]$} & {$[0.10]$} & {$[0.10]$} & {$[0.20]$} & [0.10] & 0.10 \\
\hline Housing expenditure & 49.30 & 49.60 & 67.60 & 87.30 & 107.90 & 115.10 & 148.10 & 175.80 & 228.80 & 318.20 & 134.70 \\
\hline Total expenditure $^{2}$ & 203.10 & 231.20 & 306.50 & 398.70 & 491.50 & 588.00 & 711.00 & 857.20 & 1098.30 & 1757.00 & 664.00 \\
\hline
\end{tabular}




\section{Table 2.4 Housing expenditure by age of household reference person, 2010 United Kingdom}

\begin{tabular}{|c|c|c|c|c|c|c|}
\hline & $\begin{array}{r}\text { Under } \\
30\end{array}$ & 30 to 49 & 50 to 64 & 65 to 74 & $\begin{array}{l}75 \text { or } \\
\text { over }\end{array}$ & All \\
\hline Weighted number of households (thousands) & 2,810 & 9,540 & 7,020 & 3,420 & 3,530 & 26,320 \\
\hline Total number of households in sample & 450 & 1,890 & 1,490 & 800 & 630 & 5,260 \\
\hline Total number of persons in sample & 1,060 & 5,640 & 3,200 & 1,380 & 910 & 12,180 \\
\hline Total number of adults in sample & 760 & 3,500 & 2,900 & 1,360 & 910 & 9,430 \\
\hline Weighted average number of persons per household & 2.4 & 3.0 & 2.2 & 1.8 & 1.4 & 2.3 \\
\hline Commodity or service & \multicolumn{6}{|c|}{ Average w eekly household expenditure $(£)$} \\
\hline \multicolumn{7}{|l|}{ Primary dw elling } \\
\hline Rent & 91.00 & 48.60 & 23.50 & 25.40 & 22.30 & 39.90 \\
\hline Gross rent & 91.00 & 48.60 & 23.50 & 25.40 & 22.30 & 39.90 \\
\hline less housing benefit, rebates and allowances received & 25.10 & 16.70 & 10.80 & 14.00 & 15.30 & 15.50 \\
\hline Net rent ${ }^{1}$ & 65.90 & 31.90 & 12.70 & 11.40 & 7.00 & 24.40 \\
\hline Mortgage & 35.30 & 79.70 & 42.20 & 6.00 & 1.60 & 44.90 \\
\hline Mortgage interest payments & 22.20 & 43.60 & 18.70 & 4.10 & 0.70 & 23.80 \\
\hline Mortgage protection premiums & 1.10 & 2.40 & 1.40 & {$[0.10]$} & {$[0.00]$} & 1.40 \\
\hline Capital repayment of mortgage & 12.00 & 33.70 & 22.00 & 1.80 & {$[0.80]$} & 19.70 \\
\hline Outright purchase, including deposits & {$[0.70]$} & {$[0.20]$} & {$[0.10]$} & {$[0.10]$} & - & [0.20] \\
\hline Se condary dwelling & [1.30] & 7.60 & 6.00 & [3.70] & {$[0.00]$} & 5.00 \\
\hline Rent & - & {$[0.00]$} & {$[0.10]$} & - & - & [0.00] \\
\hline Council tax, mortgage, insurance (secondary dw elling) & {$[0.80]$} & 2.70 & 1.50 & [0.70] & {$[0.00]$} & 1.60 \\
\hline Purchase of second dw elling & {$[0.50]$} & 4.80 & {$[4.40]$} & [3.00] & - & 3.40 \\
\hline Charges & 21.40 & 28.00 & 29.70 & 26.80 & 25.20 & 27.20 \\
\hline Council tax, domestic rates & 13.90 & 20.10 & 21.80 & 19.80 & 16.60 & 19.40 \\
\hline Water charges & 6.00 & 7.00 & 7.10 & 6.20 & 5.70 & 6.60 \\
\hline Other regular housing payments including service charge for ren & 1.60 & 0.80 & 0.60 & 0.90 & 2.80 & 1.10 \\
\hline Refuse collection, including skip hire & - & [0.10] & {$[0.10]$} & [0.00] & - & [0.10] \\
\hline Moving house & 1.50 & 2.30 & 1.30 & [1.50] & [0.50] & 1.60 \\
\hline Property transaction - purchase and sale & {$[0.40]$} & 0.90 & [0.60] & [0.90] & {$[0.40]$} & 0.70 \\
\hline Property transaction - sale only & {$[0.20]$} & 0.70 & {$[0.40]$} & [0.60] & - & 0.50 \\
\hline Property transaction - purchase only & {$[0.60]$} & 0.40 & {$[0.10]$} & {$[0.00]$} & {$[0.00]$} & 0.30 \\
\hline Property transaction - other payments & [0.30] & 0.30 & [0.10] & [0.00] & {$[0.00]$} & 0.20 \\
\hline Maintenance and repair of dwelling & 2.50 & 6.90 & 7.70 & 7.10 & 7.00 & 6.70 \\
\hline Central heating repairs & 0.40 & 1.20 & 1.70 & 1.80 & 1.20 & 1.30 \\
\hline House maintenance etc. & 0.90 & 3.90 & 3.30 & 3.80 & 5.10 & 3.60 \\
\hline Paint, w allpaper, timber & 1.00 & 1.10 & 1.40 & 0.60 & 0.50 & 1.00 \\
\hline Equipment hire, small materials & 0.30 & 0.70 & 1.40 & 0.80 & 0.20 & 0.80 \\
\hline Alterations and improvements to dwelling & 7.30 & 23.80 & 26.20 & 17.20 & 8.40 & 19.80 \\
\hline Central heating installation & [0.90] & 1.60 & 1.80 & {$[0.80]$} & [1.00] & 1.40 \\
\hline DIY improvements: double glazing, kitchen units, sheds etc. & [0.30] & 0.40 & 1.50 & [0.90] & {$[0.10]$} & 0.70 \\
\hline Home improvements - contracted out & 6.10 & 20.80 & 21.40 & 15.20 & 7.20 & 16.80 \\
\hline Bathroom fittings & {$[0.00]$} & 0.70 & 1.20 & {$[0.20]$} & {$[0.10]$} & 0.60 \\
\hline Purchase of materials for capital improvements & {$[0.00]$} & [0.30] & {$[0.40]$} & [0.00] & [0.00] & 0.20 \\
\hline Household insurances & 2.30 & 5.30 & 6.00 & 5.20 & 4.40 & 5.00 \\
\hline Structure & 0.90 & 2.60 & 3.00 & 2.60 & 2.10 & 2.50 \\
\hline Contents & 1.40 & 2.60 & 2.80 & 2.50 & 2.20 & 2.50 \\
\hline Household appliances & {$[0.00]$} & 0.10 & 0.10 & 0.20 & {$[0.10]$} & 0.10 \\
\hline Housing expenditure & 138.10 & 185.70 & 131.80 & 79.00 & 54.00 & 134.70 \\
\hline Total expenditure $^{2}$ & 578.00 & 850.90 & 747.90 & 437.60 & 279.40 & 664.00 \\
\hline
\end{tabular}

Note: Please see page xiii for symbols and conventions used in this report.

1 The figure included in total expenditure is net rent as opposed to gross rent.

2 This total includes all categories recorded in the LCF, including those outside the 'COICOP total expenditure. 


\section{Table 2.5 Household expenditure by UK Countries and regions, 2010}

\begin{tabular}{|c|c|c|c|c|c|c|c|}
\hline & $\begin{array}{r}\text { North } \\
\text { East }\end{array}$ & $\begin{array}{l}\text { North } \\
\text { West }\end{array}$ & $\begin{array}{r}\text { Yorks \& } \\
\text { the Humber }\end{array}$ & $\begin{array}{r}\text { East } \\
\text { Midlands }\end{array}$ & $\begin{array}{r}\text { West } \\
\text { Midlands }\end{array}$ & East & London \\
\hline Weighted number of households (thousands) & 1,190 & 3,040 & 2,280 & 1,950 & 2,270 & 2,460 & 3,010 \\
\hline Total number of households in sample & 260 & 600 & 490 & 410 & 470 & 520 & 480 \\
\hline Total number of persons in sample & 570 & 1,340 & 1,130 & 940 & 1,100 & 1,190 & 1,210 \\
\hline Total number of adults in sample & 450 & 1,050 & 860 & 740 & 850 & 940 & 890 \\
\hline Weighted average number of persons per household & 2.2 & 2.3 & 2.3 & 2.3 & 2.4 & 2.3 & 2.6 \\
\hline Commodity or service & \multicolumn{7}{|c|}{ Average w eekly household expenditure $(£)$} \\
\hline \multicolumn{8}{|l|}{ Primary dwelling } \\
\hline Rent & 35.40 & 32.60 & 32.40 & 30.10 & 34.60 & 35.60 & 82.50 \\
\hline Gross rent & 35.40 & 32.60 & 32.40 & 30.10 & 34.60 & 35.60 & 82.50 \\
\hline less housing benefit, rebates and allowances received & 19.60 & 14.10 & 13.30 & 8.20 & 15.90 & 14.00 & 27.90 \\
\hline Net rent ${ }^{1}$ & 15.80 & 18.50 & 19.20 & 21.90 & 18.70 & 21.70 & 54.60 \\
\hline Mortgage & 39.50 & 40.70 & 35.60 & 42.40 & 37.90 & 52.40 & 58.70 \\
\hline Mortgage interest payments & 18.30 & 21.30 & 18.20 & 22.80 & 17.90 & 28.20 & 33.10 \\
\hline Mortgage protection premiums & 1.60 & 1.30 & 1.40 & 1.30 & 1.10 & 1.40 & 1.50 \\
\hline Capital repayment of mortgage & 19.50 & 18.10 & 16.00 & 18.30 & 18.90 & 22.80 & 24.10 \\
\hline Outright purchase, including deposits & - & - & [0.00] & [0.10] & {$[0.50]$} & {$[0.00]$} & [0.40] \\
\hline Secondary dwelling & {$[0.90]$} & {$[1.30]$} & [1.50] & [9.40] & {$[0.60]$} & [2.40] & [4.00] \\
\hline Rent & - & - & {$[0.00]$} & - & - & - & [0.20] \\
\hline Council tax, mortgage, insurance (secondary dw elling) & [0.70] & [1.20] & [1.10] & [0.80] & {$[0.60]$} & [1.40] & [2.80] \\
\hline Purchase of second dw elling & [0.30] & [0.10] & {$[0.50]$} & [8.60] & {$[0.00]$} & [1.10] & [1.10] \\
\hline Charges & 21.40 & 26.20 & 24.00 & 27.10 & 23.70 & 31.20 & 31.50 \\
\hline Council tax, domestic rates & 15.10 & 18.00 & 16.50 & 19.90 & 16.60 & 22.30 & 22.30 \\
\hline Water charges & 6.10 & 7.20 & 6.60 & 6.80 & 6.00 & 7.30 & 6.40 \\
\hline \multicolumn{8}{|l|}{ Other regular housing payments including service charge } \\
\hline $\begin{array}{l}\text { for rent } \\
\text { Refuse collection, including skip hire }\end{array}$ & [0.20] & 0.90 & 0.90 & 0.40 & 1.10 & 1.50 & 2.70 \\
\hline Refuse collection, including skip hire & - & {$[0.20]$} & [0.10] & - & - & - & [0.20] \\
\hline Moving house & {$[1.00]$} & 1.50 & [0.60] & [1.20] & {$[1.40]$} & {$[2.60]$} & {$[1.70]$} \\
\hline Property transaction - purchase and sale & [0.70] & [1.20] & [0.00] & [0.30] & {$[0.80]$} & [0.90] & [0.50] \\
\hline Property transaction - sale only & - & [0.00] & {$[0.20]$} & {$[0.60]$} & {$[0.30]$} & [1.10] & [0.40] \\
\hline Property transaction - purchase only & [0.20] & {$[0.30]$} & {$[0.20]$} & [0.20] & {$[0.10]$} & {$[0.40]$} & [0.50] \\
\hline Property transaction - other payments & {$[0.10]$} & [0.00] & [0.20] & [0.10] & {$[0.20]$} & {$[0.20]$} & [0.30] \\
\hline Maintenance and repair of dwelling & 4.50 & 6.50 & 6.00 & 7.90 & 4.90 & 7.00 & 8.50 \\
\hline Central heating repairs & 0.50 & 1.70 & 1.20 & 1.60 & 0.90 & 1.60 & 1.30 \\
\hline House maintenance etc. & 1.70 & 3.00 & 2.40 & 3.70 & 2.80 & 3.90 & 6.00 \\
\hline Paint, w allpaper, timber & 2.00 & 0.70 & 1.20 & 1.10 & 0.70 & 1.00 & 0.60 \\
\hline Equipment hire, small materials & [0.40] & 1.20 & 1.20 & 1.50 & 0.50 & 0.60 & 0.60 \\
\hline Alterations and improvements to dwelling & 10.50 & 14.20 & 9.10 & 29.00 & 11.70 & 22.60 & 33.50 \\
\hline Central heating installation & [1.00] & {$[1.90]$} & [0.60] & [1.20] & {$[0.60]$} & [1.60] & [2.70] \\
\hline DY improvements: double glazing, kitchen units, sheds etc. & [0.40] & [0.40] & [0.40] & [0.30] & {$[0.30]$} & [1.20] & [2.20] \\
\hline Home improvements - contracted out & 8.10 & 11.20 & 4.70 & 27.30 & 9.90 & 19.30 & 27.40 \\
\hline Bathroom fittings & [0.00] & {$[0.60]$} & [2.90] & [0.20] & {$[0.70]$} & {$[0.10]$} & [1.20] \\
\hline Purchase of materials for Capital Improvements & [1.00] & {$[0.10]$} & [0.50] & [0.10] & {$[0.20]$} & {$[0.40]$} & {$[0.00]$} \\
\hline Household insurances & 4.40 & 4.80 & 4.90 & 4.90 & 4.30 & 5.20 & 6.20 \\
\hline Structure & 2.20 & 2.40 & 2.30 & 2.40 & 2.00 & 2.60 & 3.10 \\
\hline Contents & 2.20 & 2.30 & 2.40 & 2.40 & 2.30 & 2.50 & 3.00 \\
\hline Household appliances & [0.00] & {$[0.10]$} & [0.20] & [0.10] & {$[0.10]$} & [0.10] & [0.10] \\
\hline Housing expenditure & 98.00 & 113.70 & 100.90 & 143.90 & 103.70 & 145.10 & 199.00 \\
\hline Total expenditure $^{2}$ & 510.30 & 585.40 & 568.00 & 670.70 & 537.40 & 712.70 & 893.00 \\
\hline
\end{tabular}

Please see page xiii for symbols and conventions used in this report.

1 The figure included in total expenditure is net rent as opposed to gross rent.

2 This total includes all categories recorded in the LCF, including those outside the 'COICOP' total expenditure. 


\section{Table 2.5 Household expenditure by UK Countries and region, 2010} (cont.)

\begin{tabular}{|c|c|c|c|c|c|c|c|}
\hline & $\begin{array}{r}\text { South } \\
\text { East }\end{array}$ & $\begin{array}{l}\text { South } \\
\text { West }\end{array}$ & England & Wales & Scotland $\mathbf{N}$ & $\begin{array}{l}\text { Northern } \\
\text { Ireland }\end{array}$ & $\begin{array}{r}\text { United } \\
\text { Kingdom }\end{array}$ \\
\hline Weighted number of households (thousands) & 3,470 & 2,340 & 22,010 & 1,260 & 2,320 & 720 & 26,320 \\
\hline Total number of households in sample & 680 & 500 & 4,390 & 260 & 470 & 150 & 5,260 \\
\hline Total number of persons in sample & 1,560 & 1,120 & 10,160 & 620 & 1,040 & 360 & 12,180 \\
\hline Total number of adults in sample & 1,200 & 860 & 7,860 & 480 & 830 & 270 & 9,430 \\
\hline Weighted average number of persons per household & 2.4 & 2.3 & 2.3 & 2.4 & 2.2 & 2.5 & 2.3 \\
\hline Commodity or service & \multicolumn{7}{|c|}{ Average w eekly household expenditure $(£)$} \\
\hline \multicolumn{8}{|l|}{ Primary dw elling } \\
\hline Rent & 42.80 & 36.10 & 41.80 & 32.40 & 30.60 & 23.80 & 39.90 \\
\hline Gross rent & 42.80 & 36.10 & 41.80 & 32.40 & 30.60 & 23.80 & 39.90 \\
\hline less housing benefit, rebates and allowances received & 17.30 & 13.10 & 16.20 & 11.50 & 12.00 & 10.60 & 15.50 \\
\hline Net rent ${ }^{1}$ & 25.50 & 23.00 & 25.60 & 20.80 & 18.60 & 13.10 & 24.40 \\
\hline Mortgage & 51.40 & 42.60 & 45.60 & 34.90 & 44.00 & 43.30 & 44.90 \\
\hline Mortgage interest payments & 28.20 & 24.00 & 24.40 & 17.70 & 22.20 & 21.20 & 23.80 \\
\hline Mortgage protection premiums & 1.50 & 1.10 & 1.40 & 1.00 & 1.50 & 2.80 & 1.40 \\
\hline Capital repayment of mortgage & 21.60 & 17.50 & 19.90 & 16.10 & 20.30 & 19.30 & 19.70 \\
\hline Outright purchase, including deposits & {$[0.30]$} & {$[0.00]$} & {$[0.20]$} & - & {$[0.30]$} & - & [0.20] \\
\hline Secondary dwelling & 2.90 & 25.60 & 5.30 & {$[0.60]$} & [5.70] & {$[0.40]$} & 5.00 \\
\hline Rent & [0.10] & [0.00] & {$[0.00]$} & - & - & [0.20] & [0.00] \\
\hline Council tax, mortgage, insurance (secondary dw elling) & [1.90] & [4.70] & 1.80 & [0.20] & {$[0.80]$} & - & 1.60 \\
\hline Purchase of second dw elling & [0.90] & [20.80] & 3.50 & [0.40] & [4.90] & [0.20] & 3.40 \\
\hline Charges & 30.80 & 30.40 & 28.00 & 24.80 & 26.70 & 9.20 & 27.20 \\
\hline Council tax, domestic rates & 22.60 & 21.20 & 19.80 & 17.00 & 19.40 & 8.90 & 19.40 \\
\hline Water charges & 6.80 & 7.90 & 6.80 & 7.70 & 6.30 & - & 6.60 \\
\hline \multicolumn{8}{|l|}{ Other regular housing payments including service charge } \\
\hline for rent & 1.30 & 1.00 & 1.20 & [0.10] & 0.90 & 0.30 & 1.10 \\
\hline Refuse collection, including skip hire & [0.00] & [0.40] & {$[0.10]$} & - & - & - & [0.10] \\
\hline Moving house & 3.00 & [1.30] & 1.70 & {$[0.30]$} & {$[1.80]$} & {$[0.10]$} & 1.60 \\
\hline Property transaction - purchase and sale & [1.20] & [0.60] & 0.70 & - & [1.10] & [0.10] & 0.70 \\
\hline Property transaction - sale only & [1.10] & [0.40] & 0.50 & [0.00] & [0.50] & - & 0.50 \\
\hline Property transaction - purchase only & [0.30] & [0.30] & 0.30 & [0.10] & [0.20] & {$[0.00]$} & 0.30 \\
\hline Property transaction - other payments & [0.30] & [0.00] & 0.20 & [0.20] & {$[0.10]$} & - & 0.20 \\
\hline Maintenance and repair of dwelling & 7.60 & 7.50 & 6.90 & 5.20 & 6.20 & 4.40 & 6.70 \\
\hline Central heating repairs & 1.70 & 1.00 & 1.30 & 0.90 & 1.30 & 1.20 & 1.30 \\
\hline House maintenance etc. & 4.70 & 4.40 & 3.80 & 2.80 & 2.30 & 1.30 & 3.60 \\
\hline Paint, w allpaper, timber & 0.70 & 1.60 & 1.00 & [0.60] & 2.10 & [0.30] & 1.00 \\
\hline Equipment hire, small materials & 0.50 & 0.50 & 0.80 & 0.90 & 0.40 & [1.60] & 0.80 \\
\hline Alterations and improvements to dwelling & 28.80 & 17.30 & 20.70 & 21.60 & 14.00 & 5.70 & 19.80 \\
\hline Central heating installation & [1.40] & [0.90] & 1.40 & [2.00] & [0.90] & [0.30] & 1.40 \\
\hline DY improvements: double glazing, kitchen units, sheds etc. & [0.30] & [0.50] & 0.70 & [0.20] & [1.10] & [0.10] & 0.70 \\
\hline Home improvements - contracted out & 26.50 & 15.80 & 17.70 & 18.60 & 11.30 & {$[4.80]$} & 16.80 \\
\hline Bathroom fittings & [0.20] & [0.10] & 0.70 & {$[0.70]$} & {$[0.20]$} & {$[0.00]$} & 0.60 \\
\hline Purchase of materials for Capital Improvements & [0.30] & [0.00] & 0.20 & {$[0.10]$} & [0.40] & [0.40] & 0.20 \\
\hline Household insurances & 5.50 & 5.30 & 5.10 & 4.60 & 4.50 & 3.70 & 5.00 \\
\hline Structure & 2.70 & 2.60 & 2.50 & 2.20 & 2.10 & 1.80 & 2.50 \\
\hline Contents & 2.60 & 2.50 & 2.50 & 2.20 & 2.30 & 1.90 & 2.50 \\
\hline Household appliances & [2.00] & [0.10] & 0.10 & [0.20] & [0.10] & {$[0.00]$} & 0.10 \\
\hline Housing expenditure & 155.70 & 153.10 & 139.20 & 112.80 & 121.80 & 80.10 & 134.70 \\
\hline Total expenditure $^{2}$ & 739.50 & 705.50 & 675.50 & 550.60 & 639.20 & 590.30 & 664.00 \\
\hline
\end{tabular}

Please see page xiii for symbols and conventions used in this report.

1 The figure included in total expenditure is net rent as opposed to gross rent.

2 This total includes all categories recorded in the LCF, including those outside the 'COICOP' total expenditure. 


\section{Table 2.6 Housing expenditure by socio-economic classification of household reference person, 2010 United Kingdom}

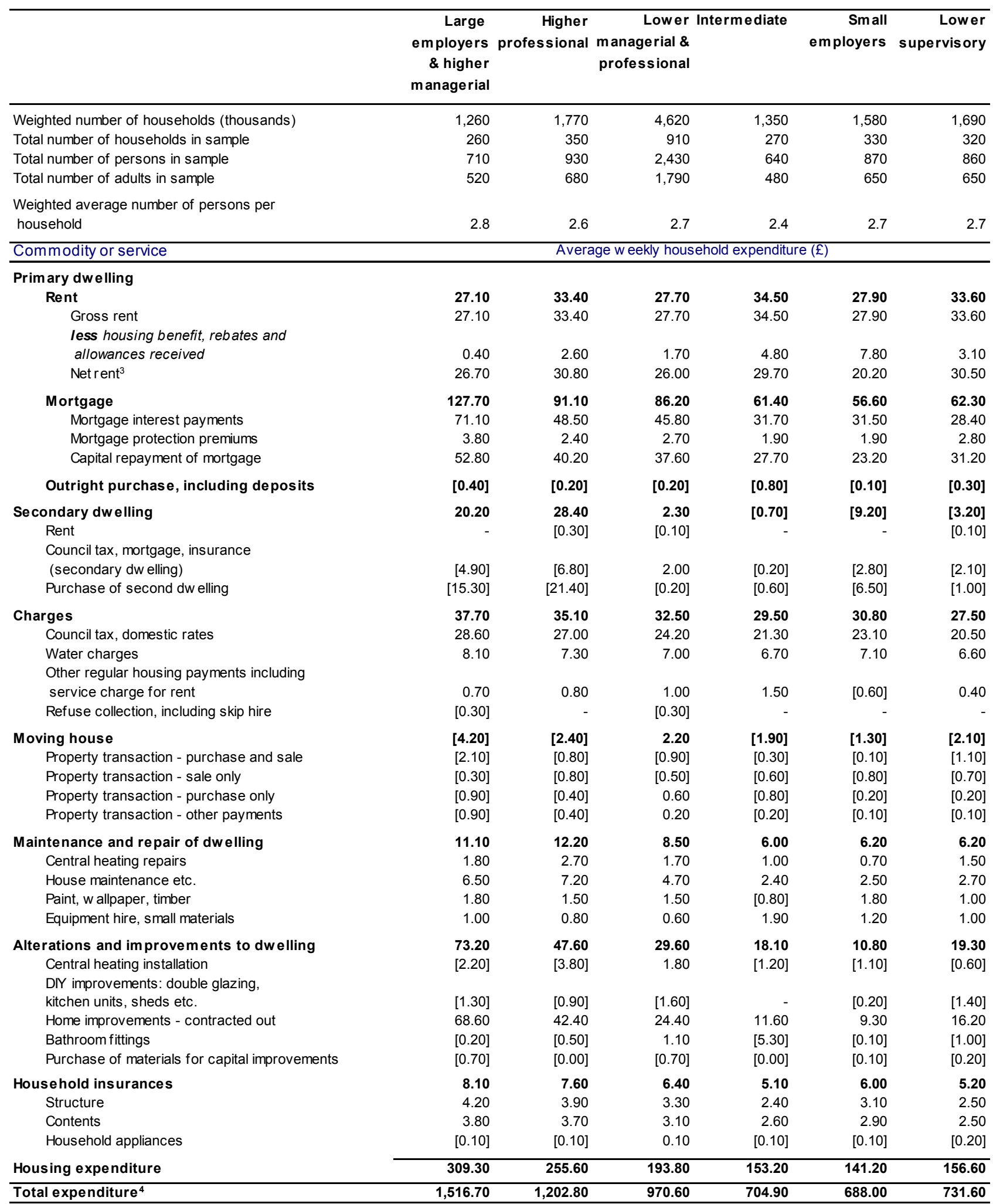

Please see page xiii for symbols and conventions used in this report.

1 Includes those w ho have never w orked.

2 Includes those $w$ ho are economically inactive.

3 The figure included in total expenditure is net rent as opposed to gross rent.

4 This total includes all categories recorded in the LCF, including those outside the 'COICOP' total expenditure. 


\section{Table 2.6 Housing expenditure by socio-economic classification of household reference person, 2010 (cont.) United Kingdom}

\begin{tabular}{|c|c|c|c|c|c|c|}
\hline & Semi-routine & Routine & $\begin{array}{r}\text { Long-term } \\
\text { unem ployed }{ }^{1}\end{array}$ & Students & $\begin{array}{r}\text { Occupation } \\
\text { not stated } \\
\& \text { not } \\
\text { classifiable }\end{array}$ & $\begin{array}{r}\text { All } \\
\text { groups }\end{array}$ \\
\hline Weighted number of households (thousands) & 1,770 & 1,520 & 490 & 590 & 9,680 & 26,320 \\
\hline Total number of households in sample & 350 & 290 & 100 & 100 & 1,990 & 5,260 \\
\hline Total number of persons in sample & 870 & 770 & 270 & 260 & 3,560 & 12,180 \\
\hline Total number of adults in sample & 650 & 590 & 150 & 180 & 3,090 & 9,430 \\
\hline $\begin{array}{l}\text { Weighted average number of persons per } \\
\text { household }\end{array}$ & 2.6 & 2.7 & 2.9 & 2.7 & 1.8 & 2.3 \\
\hline Commodity or service & \multicolumn{6}{|c|}{ Average w eekly household expenditure $(£)$} \\
\hline \multicolumn{7}{|l|}{ Prim ary dwelling } \\
\hline Rent & 54.30 & 53.80 & 104.80 & 107.40 & 40.20 & 39.90 \\
\hline $\begin{array}{l}\text { Gross rent } \\
\text { less housing benefit, rebates and }\end{array}$ & 54.30 & 53.80 & 104.80 & 107.40 & 40.20 & 39.90 \\
\hline allowances received & 11.70 & 12.50 & 83.40 & 26.80 & 28.30 & 15.50 \\
\hline Net rent ${ }^{3}$ & 42.70 & 41.30 & 21.40 & 80.50 & 11.80 & 24.40 \\
\hline Mortgage & 34.70 & 32.40 & 4.20 & 38.40 & 5.00 & 44.90 \\
\hline Mortgage interest payments & 17.50 & 15.40 & [2.50] & 23.50 & 2.90 & 23.80 \\
\hline Mortgage protection premiums & 1.00 & 1.10 & {$[0.20]$} & {$[0.60]$} & 0.10 & 1.40 \\
\hline Capital repayment of mortgage & 16.20 & 15.90 & [1.60] & 14.30 & 1.90 & 19.70 \\
\hline Outright purchase, including deposits & [0.30] & {$[0.10]$} & - & - & {$[0.00]$} & [0.20] \\
\hline $\begin{array}{l}\text { Se condary dw elling } \\
\text { Rent }\end{array}$ & [2.50] & {$[1.20]$} & {$[0.20]$} & - & $\begin{array}{l}{[1.70]} \\
{[0.00]}\end{array}$ & $\begin{array}{r}5.00 \\
{[0.00]}\end{array}$ \\
\hline $\begin{array}{l}\text { Council tax, mortgage, insurance } \\
\text { (secondary dw elling) }\end{array}$ & {$[0.10]$} & [0.90] & {$[0.20]$} & - & {$[0.40]$} & 1.60 \\
\hline Purchase of second dw elling & [2.40] & {$[0.40]$} & - & - & [1.30] & 3.40 \\
\hline Charges & 26.40 & 25.10 & 11.60 & 17.40 & 22.80 & 27.20 \\
\hline Council tax, domestic rates & 18.50 & 17.90 & 5.40 & 8.80 & 15.10 & 19.40 \\
\hline Water charges & 6.80 & 6.40 & 6.10 & 6.20 & 6.10 & 6.60 \\
\hline $\begin{array}{l}\text { Other regular housing payments including } \\
\text { service charge for rent } \\
\text { Refuse collection, including skip hire }\end{array}$ & $\begin{array}{r}0.80 \\
{[0.40]}\end{array}$ & $\begin{array}{r}{[0.80]} \\
-\end{array}$ & {$[0.20]$} & {$[2.40]$} & $\begin{array}{r}1.50 \\
{[0.00]}\end{array}$ & $\begin{array}{r}1.10 \\
{[0.10]}\end{array}$ \\
\hline Moving house & [1.60] & {$[0.30]$} & [0.40] & [1.10] & 1.10 & 1.60 \\
\hline Property transaction - purchase and sale & {$[0.80]$} & {$[0.00]$} & {$[0.20]$} & {$[0.40]$} & {$[0.60]$} & 0.70 \\
\hline Property transaction - sale only & {$[0.60]$} & - & {$[0.20]$} & - & {$[0.40]$} & 0.50 \\
\hline Property transaction - purchase only & {$[0.00]$} & {$[0.30]$} & - & {$[0.10]$} & {$[0.10]$} & 0.30 \\
\hline Property transaction - other payments & {$[0.10]$} & {$[0.00]$} & - & {$[0.60]$} & {$[0.00]$} & 0.20 \\
\hline Maintenance and repair of dwe lling & 3.20 & 2.80 & [1.30] & 3.20 & 6.20 & 6.70 \\
\hline Central heating repairs & 0.80 & 0.90 & [0.10] & [0.30] & 1.20 & 1.30 \\
\hline House maintenance etc. & 1.40 & {$[0.50]$} & {$[0.20]$} & [1.50] & 3.60 & 3.60 \\
\hline Paint, w allpaper, timber & 0.90 & 0.50 & [1.10] & {$[0.60]$} & 0.60 & 1.00 \\
\hline Equipment hire, small materials & {$[0.10]$} & {$[0.90]$} & [0.10] & {$[0.80]$} & 0.70 & 0.80 \\
\hline Alterations and improvements to dwelling & 9.20 & 7.50 & 0.80 & 8.50 & 10.30 & 19.80 \\
\hline Central heating installation & [2.20] & {$[0.70]$} & {$[0.50]$} & {$[0.50]$} & 0.80 & 1.40 \\
\hline DIY improvements: double glazing, & & & & & & \\
\hline kitchen units, sheds etc. & {$[0.60]$} & {$[0.20]$} & [0.00] & [0.00] & 0.50 & 0.70 \\
\hline Home improvements - contracted out & 6.30 & 6.20 & {$[0.30]$} & {$[8.00]$} & 8.80 & 16.80 \\
\hline Bathroom fittings & {$[0.00]$} & {$[0.20]$} & - & {$[0.00]$} & {$[0.10]$} & 0.60 \\
\hline Purchase of materials for capital improvements & {$[0.00]$} & {$[0.20]$} & - & 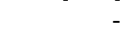 & {$[0.10]$} & 0.20 \\
\hline Household insurances & 3.40 & 3.50 & 1.10 & 2.30 & 4.10 & 5.00 \\
\hline Structure & 1.50 & 1.60 & {$[0.30]$} & 1.10 & 2.00 & 2.50 \\
\hline Contents & 1.80 & 1.90 & 0.70 & 1.20 & 2.00 & 2.50 \\
\hline Household appliances & {$[0.10]$} & {$[0.00]$} & 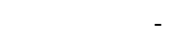 & {$[0.00]$} & 0.10 & 0.10 \\
\hline Housing expenditure & 124.00 & 114.30 & 41.10 & 151.40 & 63.00 & 134.70 \\
\hline Total expenditure ${ }^{4}$ & 515.00 & 555.30 & 226.50 & 682.00 & 351.40 & 664.00 \\
\hline
\end{tabular}

Please see page xiii for symbols and conventions used in this report.

1 Includes those who have never w orked.

2 Includes those $w$ ho are economically inactive.

3 The figure included in total expenditure is net rent as opposed to gross rent.

4 This total includes all categories recorded in the LCF, including those outside the 'COICOP' total expenditure. 
Table 2.7 Housing expenditure by household composition, 2010 United Kingdom

\begin{tabular}{|c|c|c|c|c|c|c|c|c|}
\hline & \multicolumn{2}{|c|}{ Retired hous eholds } & \multicolumn{2}{|c|}{ Non-retired } & \multicolumn{4}{|c|}{ Retired and non-retired households } \\
\hline & \multirow{2}{*}{$\begin{array}{r}\text { One } \\
\text { Person }\end{array}$} & \multirow{2}{*}{$\begin{array}{r}\text { Two } \\
\text { adults }\end{array}$} & \multirow{2}{*}{$\begin{array}{r}\text { One } \\
\text { Person }\end{array}$} & \multirow{2}{*}{$\begin{array}{r}\text { Two } \\
\text { adults }\end{array}$} & \multirow{2}{*}{$\begin{array}{r}\text { One adult } \\
\text { with } \\
\text { children }\end{array}$} & \multirow{2}{*}{$\begin{array}{r}\text { Two adults } \\
\text { with } \\
\text { children }\end{array}$} & \multicolumn{2}{|c|}{ Three or more adults } \\
\hline & & & & & & & $\begin{array}{l}\text { without } \\
\text { children }\end{array}$ & $\begin{array}{c}\text { with } \\
\text { children }\end{array}$ \\
\hline Weighted number of households (thousands) & 3,770 & 2,760 & 4,030 & 5,760 & 1,410 & 5,160 & 2,200 & $\overline{1,230}$ \\
\hline Total number of households in sample & 710 & 640 & 800 & 1,170 & 330 & 1,060 & 370 & 200 \\
\hline Total number of persons in sample & 710 & 1,270 & 800 & 2,340 & 900 & 3,990 & 1,220 & 950 \\
\hline Total number of adults in sample & 710 & 1,270 & 800 & 2,340 & 330 & 2,110 & 1,220 & 650 \\
\hline \multicolumn{9}{|l|}{ Weighted average number of persons } \\
\hline per household & 1.0 & 2.0 & 1.0 & 2.0 & 2.8 & 3.7 & 3.4 & 4.8 \\
\hline Commodity or service & \multicolumn{8}{|c|}{ Average w eekly household expenditure $(£)$} \\
\hline \multicolumn{9}{|l|}{ Primary dwelling } \\
\hline Rent & 32.30 & 14.20 & 50.50 & 36.90 & 105.30 & 38.80 & 32.30 & 43.30 \\
\hline Gross rent & 32.30 & 14.20 & 50.50 & 36.90 & 105.30 & 38.80 & 32.30 & 43.30 \\
\hline \multicolumn{9}{|l|}{ less housing benefit, rebates \& } \\
\hline allowances received & 22.70 & 8.50 & 19.90 & 3.90 & 70.80 & 14.30 & 4.00 & 10.90 \\
\hline Net rent ${ }^{1}$ & 9.70 & 5.70 & 30.50 & 33.10 & 34.50 & 24.50 & 28.20 & 32.40 \\
\hline Mortgage & 1.70 & 2.70 & 32.10 & 57.90 & 21.30 & 91.40 & 49.90 & 75.60 \\
\hline Mortgage interest payments & 1.10 & 1.30 & 17.40 & 30.00 & 12.60 & 51.10 & 22.30 & 36.10 \\
\hline Mortgage protection premiums & {$[0.00]$} & {$[0.00]$} & 0.90 & 1.70 & 0.70 & 3.00 & 2.00 & 2.10 \\
\hline Capital repayment of mortgage & {$[0.60]$} & [1.40] & 13.90 & 26.20 & 8.00 & 37.40 & 25.60 & 37.40 \\
\hline $\begin{array}{l}\text { Outright purchase, including } \\
\text { deposits }\end{array}$ & - & [0.10] & {$[0.10]$} & [0.20] & {$[0.00]$} & {$[0.10]$} & [0.50] & [0.90] \\
\hline Secondary dwelling & 0.10 & 0.50 & 1.10 & 14.20 & 0.30 & 5.30 & 6.10 & 1.30 \\
\hline Rent & - & - & {$[0.10]$} & {$[0.10]$} & {$[0.00]$} & - & {$[0.10]$} & - \\
\hline \multicolumn{9}{|l|}{ Council tax, mortgage, insurance } \\
\hline (secondary dw elling) & {$[0.10]$} & [0.50] & {$[0.80]$} & 2.90 & [0.20] & 3.30 & [0.90] & [0.30] \\
\hline Purchase of second dw elling & - & - & {$[0.20]$} & 11.20 & - & [2.00] & [5.10] & {$[1.00]$} \\
\hline Charges & 20.10 & 31.10 & 20.40 & 31.60 & 15.30 & 30.70 & 32.10 & 32.40 \\
\hline Council tax, domestic rates & 12.40 & 23.30 & 13.30 & 23.50 & 8.60 & 22.50 & 23.70 & 23.70 \\
\hline Water charges & 5.20 & 6.60 & 5.40 & 6.90 & 6.70 & 7.50 & 7.60 & 8.60 \\
\hline \multicolumn{9}{|l|}{ Other regular housing payments } \\
\hline including service charge for rent & 2.50 & 1.20 & 1.70 & 1.00 & {$[0.10]$} & 0.50 & 0.50 & {$[0.10]$} \\
\hline Refuse collection, including skip hire & - & {$[0.00]$} & {$[0.00]$} & {$[0.10]$} & - & {$[0.10]$} & [0.30] & - \\
\hline Moving house & 0.60 & 1.80 & 1.70 & 2.10 & 1.70 & 2.00 & 0.90 & 1.10 \\
\hline Property transaction - purchase and sale & {$[0.40]$} & [1.10] & {$[1.00]$} & [0.50] & {$[1.00]$} & {$[0.80]$} & {$[0.40]$} & [1.00] \\
\hline Property transaction - sale only & {$[0.20]$} & {$[0.60]$} & [0.30] & {$[0.80]$} & [0.50] & [0.50] & {$[0.20]$} & 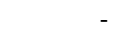 \\
\hline Property transaction - purchase only & - & {$[0.10]$} & [0.30] & 0.60 & - & {$[0.40]$} & {$[0.10]$} & - \\
\hline Property transaction - other payments & {$[0.00]$} & {$[0.00]$} & [0.10] & 0.20 & [0.10] & 0.40 & {$[0.20]$} & [0.10] \\
\hline Maintenance and repair of dwelling & 5.40 & 8.60 & 3.50 & 8.30 & 2.30 & 8.50 & 6.60 & 6.60 \\
\hline Central heating repairs & 1.10 & 1.90 & 0.70 & 1.50 & 1.10 & 1.30 & 1.80 & 1.20 \\
\hline House maintenance etc. & 3.80 & 5.10 & 2.10 & 4.00 & 0.60 & 4.80 & 2.80 & 2.20 \\
\hline Paint, w allpaper, timber & 0.40 & 0.60 & 0.50 & 1.30 & {$[0.50]$} & 1.40 & 1.20 & 2.80 \\
\hline Equipment hire, small materials & {$[0.10]$} & 1.00 & 0.20 & 1.50 & [0.10] & 1.00 & 0.70 & [0.50] \\
\hline Alterations and improvements to dwelling & 5.40 & 17.50 & 11.20 & 28.10 & 4.00 & 35.70 & 15.70 & 16.60 \\
\hline Central heating installation & [0.90] & [0.90] & 1.40 & 1.70 & [0.50] & 2.10 & [1.20] & [0.50] \\
\hline \multicolumn{9}{|l|}{ DYY improvements: double glazing, } \\
\hline kitchen units, sheds etc. & {$[0.00]$} & {$[1.00]$} & [1.70] & 1.00 & {$[0.20]$} & [0.40] & {$[0.60]$} & \\
\hline Home improvements - contracted out & 4.30 & 15.50 & 7.90 & 22.80 & [3.20] & 32.30 & 13.60 & 15.90 \\
\hline Bathroom fittings & {$[0.10]$} & {$[0.10]$} & [0.10] & 2.00 & {$[0.10]$} & {$[0.60]$} & {$[0.30]$} & [0.20] \\
\hline Purchase of materials for & & & & & & & & \\
\hline capital improvements & {$[0.00]$} & {$[0.00]$} & {$[0.00]$} & {$[0.70]$} & {$[0.10]$} & {$[0.40]$} & {$[0.00]$} & {$[0.10]$} \\
\hline Household insurances & 3.70 & 5.60 & 3.50 & 5.70 & 2.60 & 5.90 & 6.10 & 6.50 \\
\hline Structure & 1.70 & 2.80 & 1.80 & 2.80 & 1.00 & 2.90 & 3.20 & 3.30 \\
\hline Contents & 1.90 & 2.70 & 1.80 & 2.90 & 1.50 & 2.90 & 2.70 & 3.20 \\
\hline Household appliances & {$[0.10]$} & {$[0.10]$} & {$[0.00]$} & 0.10 & [0.10] & [0.10] & {$[0.20]$} & {$[0.10]$} \\
\hline Housing expenditure & 46.60 & 73.60 & 104.20 & 181.20 & 82.00 & 204.10 & 146.10 & 173.50 \\
\hline Total expenditure $^{2}$ & 230.20 & 441.60 & 411.80 & 867.60 & 358.70 & 949.40 & 946.70 & $\overline{1011.30}$ \\
\hline
\end{tabular}

Note: Please see page xiii for symbols and conventions used in this report.

1 The figure included in total expenditure is net rent as opposed to gross rent.

2 This total includes all categories recorded in the LCF, including those outside the 'COICOP' total expenditure. 


\section{Table 2.8 Expenditure on rent ${ }^{1}$ by renters, 2008 to 2010 United Kingdom}

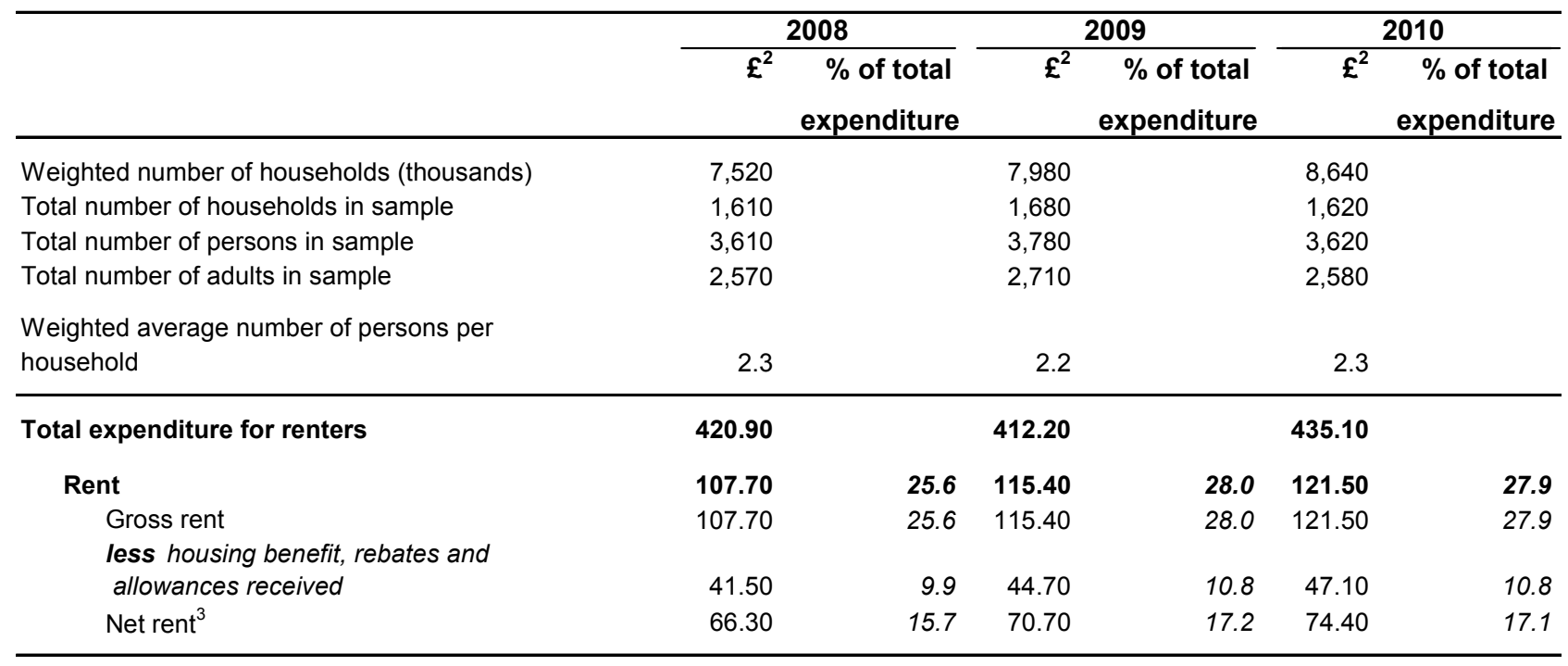

Note: Please see page xiii for symbols and conventions used in this report.

1 Primary dwelling.

2 Average weekly household expenditure (£).

3 The figure included in total expenditure is net rent as opposed to gross rent.

\section{Table 2.9 Expenditure on mortgages ${ }^{1}$ by mortgage holders, 2008 to 2010 \\ United Kingdom}

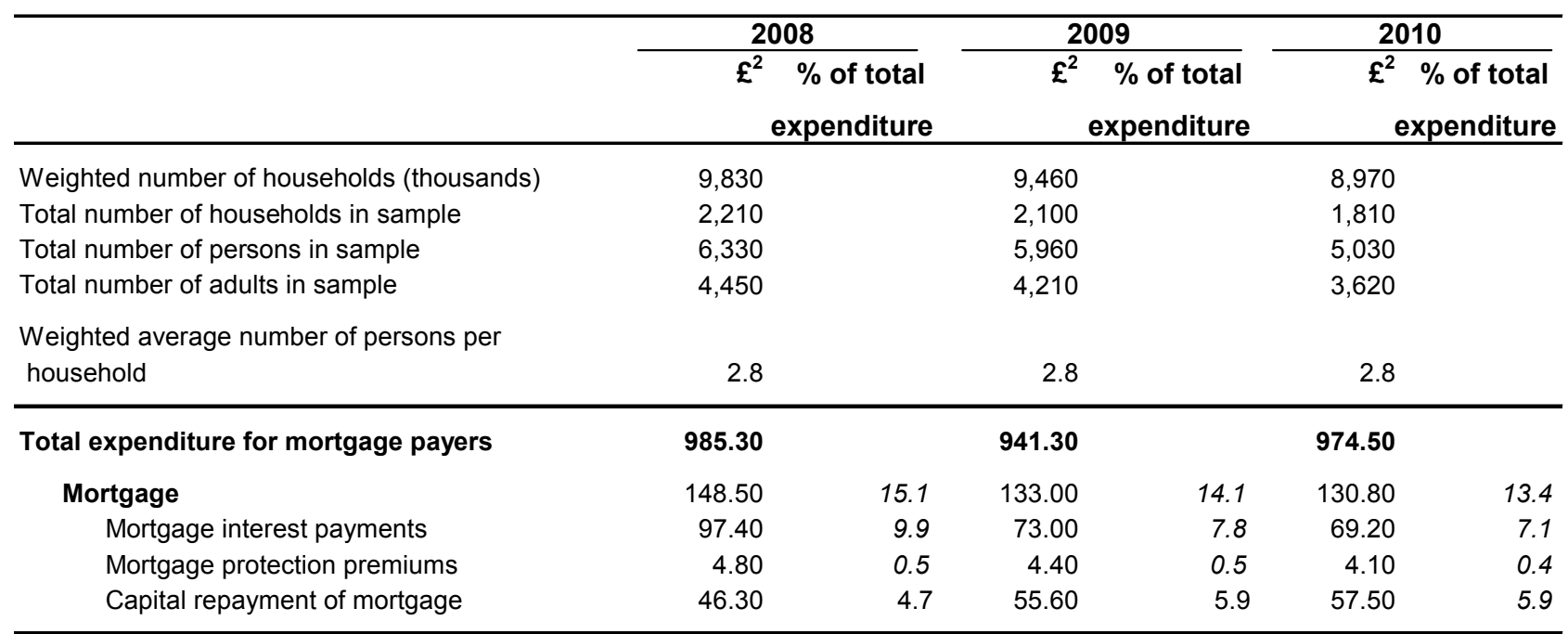

Note: Please see page xiii for symbols and conventions used in this report.

1 Primary dwelling.

2 Average weekly household expenditure (£). 


\section{Table 2.10 Expenditure on rent and mortgages ${ }^{1}$ by renters and mortgage holders by gross income decile group, 2010 United Kingdom}

\begin{tabular}{|c|c|c|c|c|c|c|c|c|c|c|c|}
\hline & \multicolumn{11}{|c|}{ Gross income decile group } \\
\hline & 1 & 2 & 3 & 4 & 5 & 6 & 7 & 8 & 9 & 10 & All \\
\hline Weighted number of households (thousands) & 1,670 & 1,360 & 1,070 & 1,010 & 910 & 730 & 690 & 570 & 340 & 310 & 8,640 \\
\hline Total number of households in sample & 320 & 270 & 210 & 200 & 160 & 130 & 120 & 100 & 60 & 50 & 1,620 \\
\hline Total number of persons in sample & 440 & 520 & 420 & 470 & 480 & 380 & 340 & 270 & 170 & 150 & 3,620 \\
\hline Total number of adults in sample & 340 & 350 & 300 & 310 & 300 & 260 & 260 & 210 & 140 & 120 & 2,580 \\
\hline \multicolumn{12}{|l|}{ Weighted average number of persons } \\
\hline per household & 1.3 & 1.9 & 2.0 & 2.4 & 2.8 & 2.8 & 2.8 & 2.8 & 3.0 & 3.4 & 2.3 \\
\hline Commodity or service & \multicolumn{11}{|c|}{ Average w eekly household expenditure $(£)$} \\
\hline Rent for renters & 113.60 & 112.90 & 114.50 & 114.20 & 112.90 & 104.10 & 121.00 & 137.60 & 152.20 & 255.40 & 121.50 \\
\hline Gross rent & 113.60 & 112.90 & 114.50 & 114.20 & 112.90 & 104.10 & 121.00 & 137.60 & 152.20 & 255.40 & 121.50 \\
\hline \multicolumn{12}{|l|}{ less housing benefit, rebates and } \\
\hline allowances received & 81.90 & 77.20 & 68.00 & 44.60 & 33.30 & 11.90 & [9.40] & {$[2.30]$} & [5.40] & {$[0.30]$} & 47.10 \\
\hline Net rent ${ }^{2}$ & 31.70 & 35.60 & 46.60 & 69.60 & 79.60 & 92.20 & 111.60 & 135.30 & 146.80 & 255.20 & 74.40 \\
\hline Weighted number of households (thousands) & 230 & 200 & 300 & 530 & 680 & 930 & 1,240 & 1,470 & 1,680 & 1,720 & 8,970 \\
\hline Total number of households in sample & 50 & 40 & 70 & 110 & 150 & 190 & 250 & 290 & 330 & 330 & 1,810 \\
\hline Total number of persons in sample & 70 & 70 & 150 & 220 & 350 & 480 & 710 & 840 & 1,040 & 1,090 & 5,030 \\
\hline Total number of adults in sample & 60 & 60 & 100 & 170 & 250 & 350 & 520 & 600 & 740 & 780 & 3,620 \\
\hline \multicolumn{12}{|l|}{ Weighted average number of persons } \\
\hline per household & 1.4 & 1.7 & 2.1 & 2.0 & 2.4 & 2.6 & 2.9 & 2.9 & 3.1 & 3.2 & 2.8 \\
\hline Commodity or service & \multicolumn{11}{|c|}{ Average w eekly household expenditure $(£)$} \\
\hline Mortgage for mortgage holders & 84.00 & 70.40 & 75.30 & 87.80 & 103.70 & 95.40 & 113.40 & 121.20 & 151.80 & 197.20 & 130.80 \\
\hline Mortgage interest payments & 57.10 & 36.20 & 39.90 & 50.50 & 48.30 & 50.50 & 58.90 & 62.70 & 81.00 & 105.50 & 69.20 \\
\hline Mortgage protection premiums & {$[2.20]$} & [1.70] & 3.20 & 2.10 & 2.90 & 3.80 & 3.30 & 4.60 & 5.00 & 5.20 & 4.10 \\
\hline Capital repayment of mortgage & 24.70 & 32.50 & 32.20 & 35.20 & 52.60 & 41.10 & 51.10 & 53.90 & 65.80 & 86.50 & 57.50 \\
\hline
\end{tabular}

Note: Please see page xiii for symbols and conventions used in this report.

1 Primary dw elling.

2 The figure included in total expenditure is net rent as opposed to gross rent. 


\section{Table 2.11 Expenditure on rent and mortgages ${ }^{1}$ by renters and mortgage holders by UK Countries and region, 2010}

\begin{tabular}{|c|c|c|c|c|c|c|c|}
\hline & $\begin{array}{l}\text { North } \\
\text { East }\end{array}$ & $\begin{array}{l}\text { North } \\
\text { West }\end{array}$ & $\begin{array}{c}\text { Yorks \& } \\
\text { the Humber }\end{array}$ & $\begin{array}{r}\text { East } \\
\text { Midlands }\end{array}$ & $\begin{array}{l}\text { West } \\
\text { Midlands }\end{array}$ & East & London \\
\hline Weighted number of households (thousands) & 460 & 950 & 750 & 590 & 720 & 740 & 1,240 \\
\hline Total number of households in sample & 90 & 180 & 150 & 110 & 140 & 150 & 190 \\
\hline Total number of persons in sample & 190 & 370 & 330 & 250 & 310 & 330 & 490 \\
\hline Total number of adults in sample & 150 & 270 & 230 & 190 & 220 & 250 & 330 \\
\hline Weighted average number of persons per household & 2.1 & 2.1 & 2.1 & 2.3 & 2.3 & 2.2 & 2.7 \\
\hline Commodity or service & \multicolumn{7}{|c|}{ Average w eekly hous ehold expenditure $(£)$} \\
\hline Rent by renters & 92.00 & 104.50 & 98.30 & 99.70 & 109.00 & 118.00 & 199.90 \\
\hline Gross rent & 92.00 & 104.50 & 98.30 & 99.70 & 109.00 & 118.00 & 199.90 \\
\hline less housing benefit, rebates and allowances received & 51.00 & 45.20 & 40.20 & 27.20 & 50.20 & 46.20 & 67.60 \\
\hline Net rent ${ }^{2}$ & 41.00 & 59.30 & 58.10 & 72.50 & 58.80 & 71.70 & 132.30 \\
\hline Weighted number of households (thousands) & 450 & 1,060 & 770 & 660 & 780 & 840 & 960 \\
\hline Total number of households in sample & 100 & 200 & 170 & 150 & 160 & 170 & 150 \\
\hline Total number of persons in sample & 250 & 570 & 480 & 400 & 460 & 490 & 420 \\
\hline Total number of adults in sample & 190 & 410 & 340 & 290 & 330 & 350 & 310 \\
\hline Weighted average number of persons per household & 2.6 & 2.8 & 2.8 & 2.7 & 2.8 & 2.8 & 2.7 \\
\hline Commodity or service & \multicolumn{7}{|c|}{ Average w eekly household expenditure $(£)$} \\
\hline Mortgage by mortgage holders & 102.70 & 115.50 & 105.80 & 124.20 & 108.80 & 153.80 & 180.90 \\
\hline Mortgage interest payments & 47.30 & 60.30 & 54.10 & 66.80 & 51.30 & 82.80 & 101.90 \\
\hline Mortgage protection premiums & 4.30 & 3.80 & 4.20 & 3.80 & 3.20 & 4.10 & 4.70 \\
\hline \multirow[t]{2}{*}{ Capital repayment of mortgage } & 51.10 & 51.40 & 47.50 & 53.60 & 54.30 & 67.00 & 74.30 \\
\hline & $\begin{array}{r}\text { South } \\
\text { East }\end{array}$ & $\begin{array}{l}\text { South } \\
\text { West }\end{array}$ & England & Wales & Scotland & $\begin{array}{r}\text { Northern } \\
\text { Ireland }\end{array}$ & $\begin{array}{r}\text { United } \\
\text { Kingdom }\end{array}$ \\
\hline Weighted number of households (thousands) & 1,080 & 740 & 7,270 & 360 & 810 & 200 & 8,640 \\
\hline Total number of households in sample & 200 & 150 & 1,350 & 70 & 150 & 40 & 1,620 \\
\hline Total number of persons in sample & 460 & 330 & 3,060 & 180 & 300 & 80 & 3,620 \\
\hline Total number of adults in sample & 310 & 220 & 2,160 & 120 & 240 & 60 & 2,580 \\
\hline Weighted average number of persons per household & 2.4 & 2.1 & 2.3 & 2.4 & 1.9 & 2.0 & 2.3 \\
\hline Commodity or service & \multicolumn{7}{|c|}{ Average w eekly household expenditure $(£)$} \\
\hline Rent by renters & 138.20 & 113.40 & 126.70 & 112.60 & 88.00 & 85.80 & 121.50 \\
\hline Gross rent & 138.20 & 113.40 & 126.70 & 112.60 & 88.00 & 85.80 & 121.50 \\
\hline less housing benefit, rebates and allowances received & 55.80 & 41.00 & 49.10 & 40.10 & 34.40 & 38.40 & 47.10 \\
\hline Net rent ${ }^{2}$ & 82.40 & 72.40 & 77.50 & 72.50 & 53.60 & 47.40 & 74.40 \\
\hline Weighted number of households (thousands) & 1,170 & 730 & 7,420 & 420 & 880 & 260 & 8,970 \\
\hline Total number of households in sample & 230 & 160 & 1,490 & 90 & 180 & 50 & 1,810 \\
\hline Total number of persons in sample & 650 & 450 & 4,150 & 240 & 480 & 160 & 5,030 \\
\hline Total number of adults in sample & 470 & 320 & 2,990 & 180 & 350 & 100 & 3,620 \\
\hline Weighted average number of persons per household & 2.9 & 2.9 & 2.8 & 2.7 & 2.7 & 3.1 & 2.8 \\
\hline Commodity or service & \multicolumn{7}{|c|}{ Average w eekly household expenditure $(£)$} \\
\hline Mortgage by mortgage holders & 151.80 & 135.20 & 134.20 & 105.40 & 116.70 & 122.10 & 130.80 \\
\hline Mortgage interest payments & 83.50 & 76.20 & 71.70 & 53.60 & 58.80 & 59.80 & 69.20 \\
\hline Mortgage protection premiums & 4.50 & 3.40 & 4.00 & 3.10 & 4.00 & 7.90 & 4.10 \\
\hline Capital repayment of mortgage & 63.70 & 55.60 & 58.60 & 48.70 & 53.80 & 54.40 & 57.50 \\
\hline
\end{tabular}

Note: Please see page xiii for symbols and conventions used in this report.

1 Primary dw elling.

2 The figure included in total expenditure is net rent as opposed to gross rent. 